\title{
MicroRNA-208a is a regulator of cardiac hypertrophy and conduction in mice
}

\author{
Thomas E. Callis,, ${ }^{1,2}$ Kumar Pandya, ${ }^{3}$ Hee Young Seok, ${ }^{1,2}$ Ru-Hang Tang,, ${ }^{1,2,4}$ Mariko Tatsuguchi, ${ }^{1,2}$ \\ Zhan-Peng Huang, ${ }^{1,2}$ Jian-Fu Chen,, ${ }^{1,2}$ Zhongliang Deng, ${ }^{1,5}$ Bronwyn Gunn, ${ }^{1}$ Janelle Shumate,1 \\ Monte S. Willis,,$^{1,3}$ Craig H. Selzman, ${ }^{1,4}$ and Da-Zhi Wang ${ }^{1,2}$
${ }^{1}$ Carolina Cardiovascular Biology Center, ${ }^{2}$ Department of Cell and Developmental Biology, ${ }^{3}$ Department of Pathology and Laboratory Medicine, and ${ }^{4}$ Department of Surgery, University of North Carolina, Chapel Hill, North Carolina, USA. \\ 5Department of Orthopaedic Surgery, The Second Affiliated Hospital, Chongqing Medical University, Chongqing, People's Republic of China.
}

\begin{abstract}
MicroRNAs (miRNAs) are a class of small noncoding RNAs that have gained status as important regulators of gene expression. Here, we investigated the function and molecular mechanisms of the miR-208 family of miRNAs in adult mouse heart physiology. We found that miR-208a, which is encoded within an intron of $\alpha$-cardiac muscle myosin heavy chain gene (Myb6), was actually a member of a miRNA family that also included miR-208b, which was determined to be encoded within an intron of $\beta$-cardiac muscle myosin heavy chain gene (Myb7). These miRNAs were differentially expressed in the mouse heart, paralleling the expression of their host genes. Transgenic overexpression of miR-208a in the heart was sufficient to induce hypertrophic growth in mice, which resulted in pronounced repression of the miR-208 regulatory targets thyroid hormone-associated protein 1 and myostatin, 2 negative regulators of muscle growth and hypertrophy. Studies of the miR-208a $\mathrm{Tg}$ mice indicated that miR-208a expression was sufficient to induce arrhythmias. Furthermore, analysis of mice lacking miR-208a indicated that miR-208a was required for proper cardiac conduction and expression of the cardiac transcription factors homeodomain-only protein and GATA4 and the gap junction protein connexin 40. Together, our studies uncover what we believe are novel miRNA-dependent mechanisms that modulate cardiac hypertrophy and electrical conduction.
\end{abstract}

\section{Introduction}

MicroRNAs (miRNAs) are an evolutionarily conserved class of small noncoding RNAs that have recently gained status as important regulators of gene expression in diverse biological processes including cellular proliferation, differentiation, and tumorigenesis (1). miRNAs are generally regarded as negative regulators of gene expression that inhibit translation and/or promote mRNA degradation by base pairing to complementary sequences within the $3^{\prime}$ untranslated region ( $3^{\prime}$ UTR) of protein-coding mRNA transcripts (2-4). miRNAs provide an additional posttranscriptional layer of spatial and temporal control of developmental and homeostatic events by altering levels of critical regulators within complex genetic pathways (5-7).

The role of miRNAs in cardiac development and function has begun to be uncovered (8-10). In the developing mouse heart, overexpression of miR-1 caused defective ventricular myocyte proliferation (11), while introduction of miR-1 into developing Xenopus embryos also interfered with heart development (12). Targeted deletion of the mouse Mir1-2 gene, 1 of the 2 miR-1 genes expressed in skeletal and cardiac muscle, leads to lethality of approximately half of Mir1-2-null animals by weaning age and some suffer from abnormal cardiac morphogenesis and cardiac conduction defects (13). The global role of miRNA function in the heart has been addressed by conditionally inhibiting miRNA maturation in the murine heart and has revealed that miRNAs play an essential role during development $(13,14)$. In addition, miRNA expression profiling studies demonstrate that the expression of specific miRNAs changes in diseased human hearts, pointing to the involvement of miRNAs in cardiomyopathies $(15,16)$.

Conflict of interest: The authors have declared that no conflict of interest exists. Citation for this article: J. Clin. Invest. 119:2772-2786 (2009). doi:10.1172/JCI36154.
A requirement for miR-208a in stress-dependent cardiac growth was recently reported (17). miR-208a is encoded by an intron of the $\alpha$-cardiac muscle myosin heavy chain gene (Myb6) and is expressed specifically in the heart. Here, we report that miR-208a belongs to a miRNA family that includes miR-208b, which is encoded within the $\beta$-cardiac muscle myosin heavy chain gene (Myb7). We show that expression of miR-208a and miR-208b is developmentally and pathologically regulated in the heart. We found that the adult isoform miR-208a was sufficient to induce cardiac remodeling and regulate the expression of hypertrophy pathway components, including upregulation of $\beta \mathrm{MHC}$. Conversely, genetic deletion of miR-208a resulted in decreased $\beta \mathrm{MHC}$ expression. We found that miR-208a and miR-208b shared nearly identical sequence identity and were able to repress the same target genes. Furthermore, miR-208a was sufficient to induce arrhythmias and miR-208a genetic deletion resulted in aberrant cardiac conduction. We also found miR-208a was required to maintain expression of cardiac transcription factors known to be important for development of the conduction system. Our results clearly demonstrate the pathological consequences of perturbing the expression of a single miRNA in the heart and establish miR-208a as a regulator of hypertrophic growth and the cardiac conduction system.

\section{Results}

Cardiac myosin beavy chain genes encode intronic miRNAs. During development, the $\alpha \mathrm{MHC}$ and $\beta \mathrm{MHC}$ isoforms are expressed in a developmental stage-specific manner (18). In mouse hearts, the slower isoform $\beta \mathrm{MHC}$ is fetal specific, while the faster isoform $\alpha \mathrm{MHC}$ becomes the predominant isoform in the adult heart. In humans and other large mammals, $\beta$ MHC expression continues into adulthood. However, increased $\beta$ MHC expression is a com- 
A

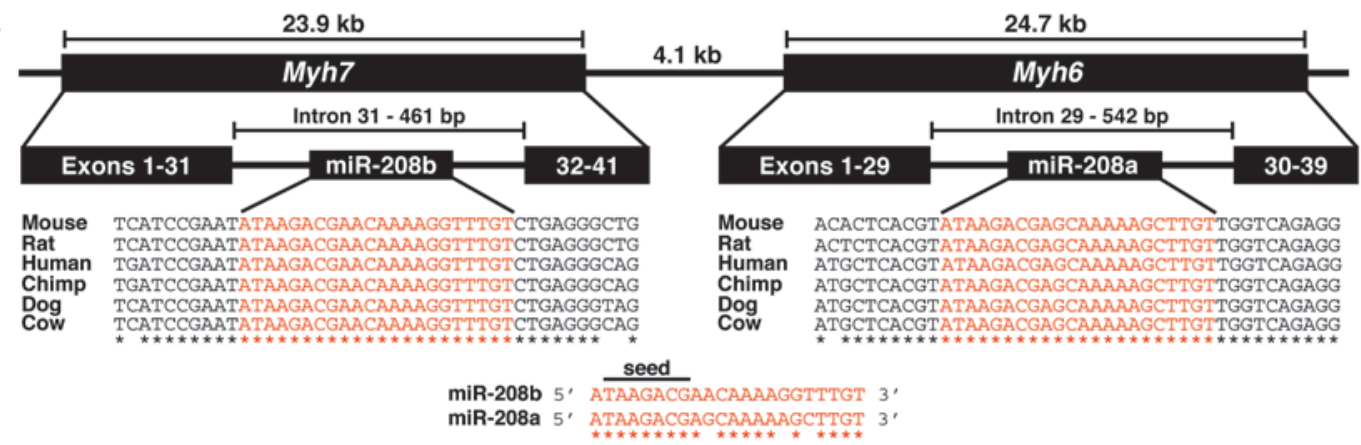

B

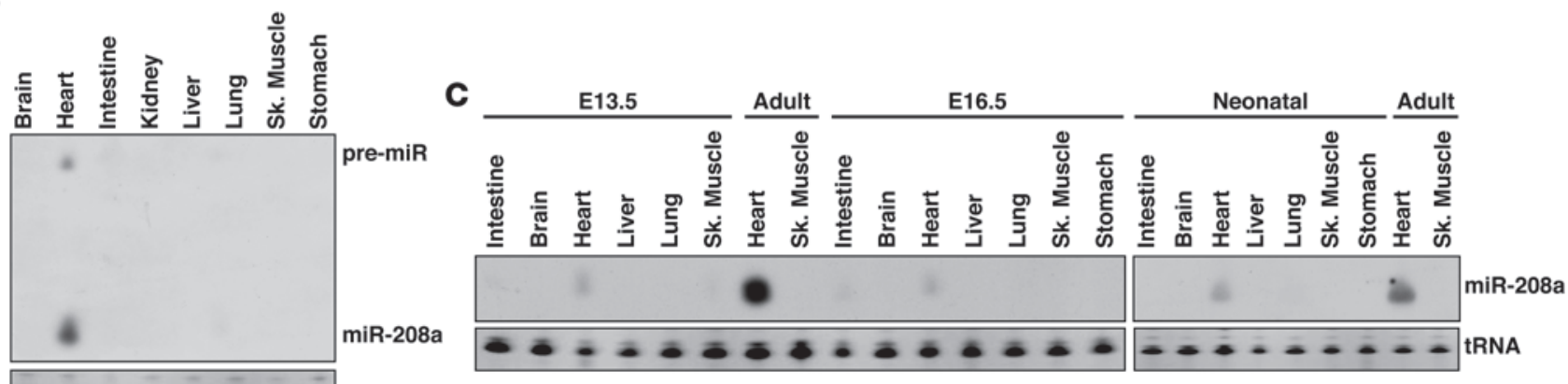

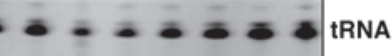

D
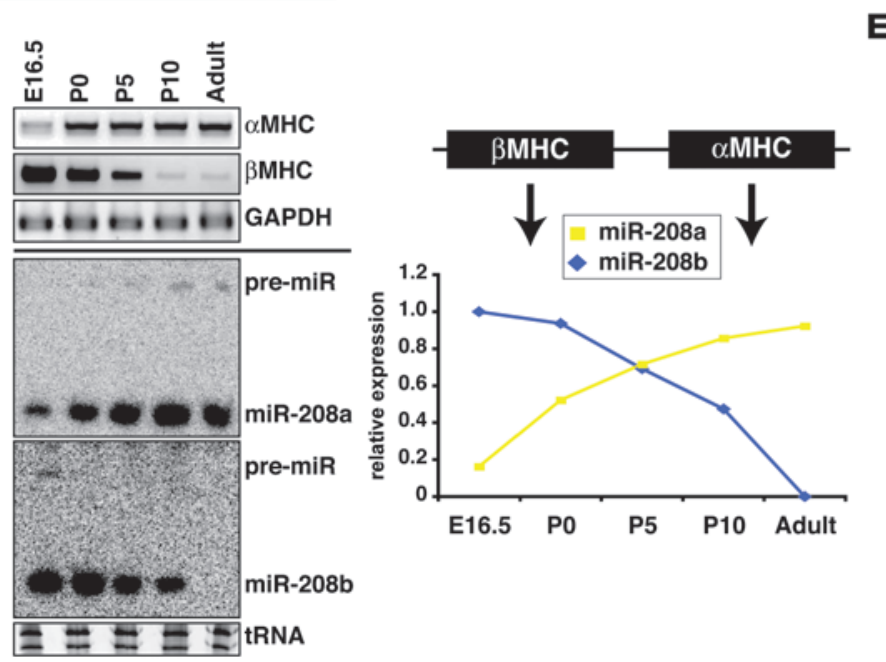

E

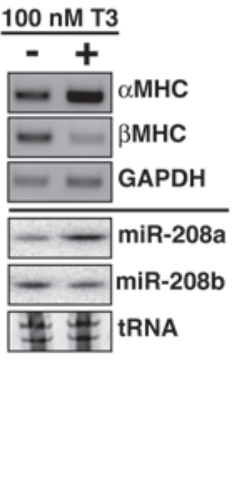

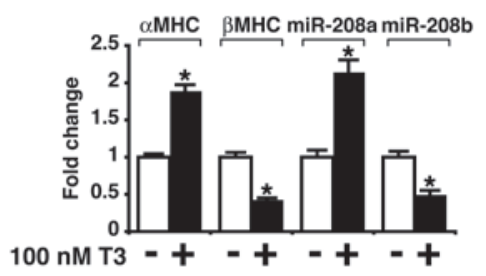

$100 \mathrm{nMT3}=+-+-+-+$

\section{Figure 1}

Expression of miR-208a and miR-208b parallels the expression of their respective host genes Myh6 and Myh7. (A) miR-208a is encoded by intron 29 of the Myh6 gene, while miR-208b is encoded by intron 31 of the Myh7 gene. miR-208a and miR-208b are highly conserved and share similar sequence identity (indicated by asterisks). (B) Detection of mature and precursor miR-208a in adult mouse tissues. Sk. muscle, skeletal muscle; tRNA, transfer RNA. (C) Detection of mature and precursor miR-208a in E13.5, E16.5, and neonatal tissues using Northern blot analysis. (D) Top left: $\alpha \mathrm{MHC}$ and $\beta \mathrm{MHC}$ transcripts were detected in E16.5, P0, P5, P10, and adult mouse hearts using RT-PCR. Bottom left: miR-208a and miR-208b expression was detected in the samples using Northern analysis. Right: Relative levels of miR-208a and miR-208b during heart development (E) Top left: $\alpha \mathrm{MHC}$ and $\beta \mathrm{MHC}$ transcripts were detected using RT-PCR in isolated rat neonatal cardiomyocytes following treatment with thyroid hormone (T3). Bottom left: miR-208a and miR-208b were detected using Northern analysis. Right: Quantitative analysis. Fold change is relative to no T3 treatment (which was set at 1 ). ${ }^{*} P<0.01$, compared with no T3 treatment.

mon feature of cardiac hypertrophy and heart failure in both mouse and human hearts $(19,20)$. An intron from each of the Myb6 and Myb7 genes host a conserved miRNA - miR-208a and miR-208b, respectively (Figure 1A). miR-208a expression was detected specifically in the adult mouse heart (Figure 1B) and could be detected at very low levels in the heart as early as E13.5 (Figure 1C). The switch from fetal isoform $\beta \mathrm{MHC}$ to the adult isoform $\alpha \mathrm{MHC}$ in the mouse occurs shortly after birth (Figure 1D). We found that a similar switch from miR-208b to miR-208a expression also occurs, suggesting they are cotranscribed with their MHC host genes (Figure 1D). miR-208a and miR-208b are of similar sequence with identical seed regions (Figure 1A), which suggests they might be functionally redundant (21). However, miR-208b was not detectable in the adult heart, indicating 
A Control miR-208a Tg

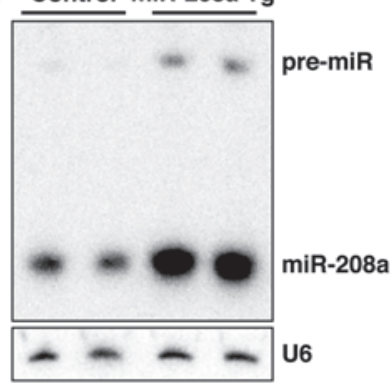

E

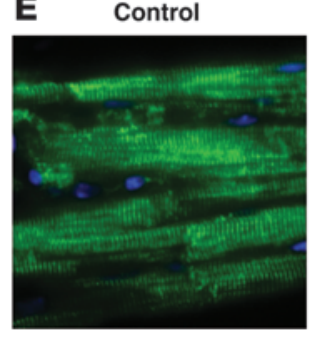

miR-208a Tg

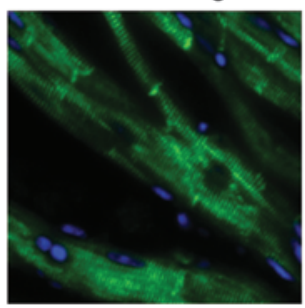

G

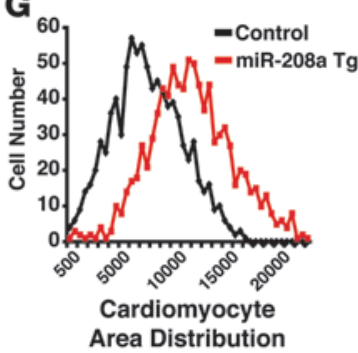

B
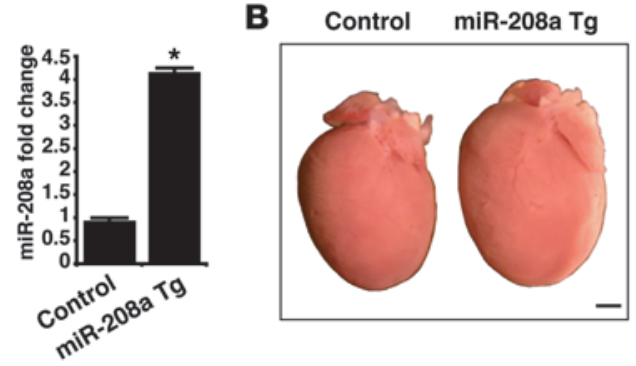

C
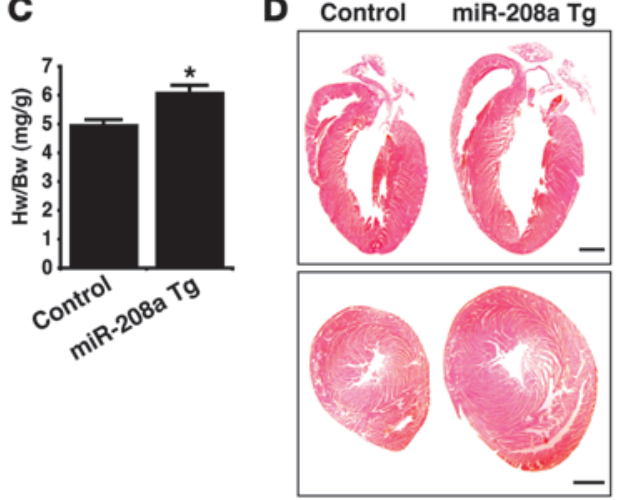

F
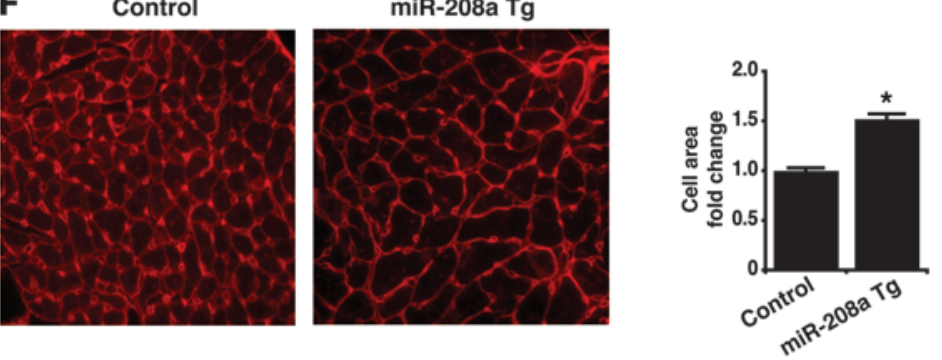

H

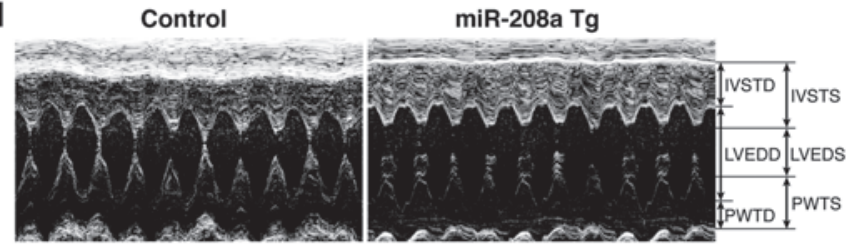

Figure 2

Hearts of miR-208a Tg mice undergo hypertrophic growth. (A) Left: Northern blot analysis showing an approximately 4-fold increase of miR-208a expression in hearts of miR-208a Tg animals compared with control littermates. U6 served as loading control. Right: Quantitative analysis of fold change in miR-208a expression compared with control animals. ${ }^{\star} P<0.001$. (B) Gross morphology of miR-208a Tg hearts was enlarged compared with control hearts. Scale bar: $1 \mathrm{~mm}$. (C) Heart weight to body weight ratios (Hw/Bw) of 4-month-old miR-208a Tg mice $(n=22)$ were significantly higher than controls $(n=19)\left(P<7 \times 10^{-7}\right)$. (D) Macroscopic view of H\&E-stained histological sections (upper, sagittal; lower, transverse) from control and miR-208a Tg hearts. Scale bars: $1 \mathrm{~mm}$. (E) Sarcomeric structure of cardiomyocytes visualized by desmin staining of histological sections. Original magnification, $\times 200$. (F) Histological sections were stained with wheat germ agglutinin-TRITC conjugate to determine cell size. Mean cardiomyocyte size of miR-208a Tg hearts $(n=930)$ was significantly larger than control cardiomyocytes $(n=926)$ $\left({ }^{\star} P<9 \times 10^{-50}\right)$. Original magnification, $\times 200$. (G) Distribution of control and miR-208a Tg cardiomyocyte cell area measurements were compared. (H) Representative M-mode echocardiographs from conscious control and miR-208a Tg mice. IVSTD, interventricular septal thickness in diastole; IVSTS, interventricular septal thickness in systole; PWTD, posterior wall thickness in diastole; PWTS, posterior wall thickness in systole; LVEDD, left ventricular end-diastolic dimension; LVESD, left ventricular end-systolic dimension.

that if miR-208a and miR-208b target the same mRNAs, they do so at different developmental stages.

Thyroid hormone signaling is a well-known regulator of $\alpha \mathrm{MHC}$ and $\beta$ MHC transcription (22). A surge of circulating thyroid hormone that occurs shortly after birth represses $\beta \mathrm{MHC}$ expression and activates $\alpha \mathrm{MHC}$ expression though negative and positive cis-acting elements within their respective promoters. We treated isolated rat cardiomyocytes with thyroid hormone and observed reduced $\beta \mathrm{MHC} / \mathrm{miR}-208 \mathrm{~b}$ expression while dramatically inducing $\alpha \mathrm{MHC} / \mathrm{miR}-208 \mathrm{a}$ expression (Figure $1 \mathrm{E}$ ). Together, those data suggest that the intronic miR-208 family and their MHC host genes are co-expressed and regulated by common transcriptional events and signaling pathways.
Cardiac overexpression of $m i R-208 a$ is sufficient to induce cardiac bypertrophy. In an effort to understand the function of miR-208a in the adult heart, we overexpressed miR-208a specifically in the heart under the control of the $\alpha$-myosin heavy chain $(\alpha M H C)$ promoter using a bigenic system. An advantage of this strategy is that miR-208a is overexpressed specifically at the same time and place it would normally be expressed. The overexpression strategy consisted of a transgene encoding miR-208a downstream of a tetracycline-responsive promoter (TRE-miR-208a) and a second transgene encoding the tetracycline-controlled transactivator protein driven by the $\alpha M H C$ promoter $(\alpha M H C-t T A)(23)$. Using this system, we found that cardiac-specific overexpression of miR-208a did not cause embryonic lethality, and thus administration of tet- 


\section{Table 1}

Echocardiography of dimensions and function of miR-208a Tg mice

\begin{tabular}{lcc} 
Parameter & Control & miR-208a Tg \\
BW $(\mathrm{g})$ & $25.0 \pm 1.14$ & $26.1 \pm 1.24$ \\
LV mass $(\mathrm{mg})$ & $107 \pm 1.45$ & $163 \pm 6.33^{\mathrm{A}}$ \\
LV mass/BW $(\mathrm{mg} / \mathrm{g})$ & $3.94 \pm 0.09$ & $6.04 \pm 0.24^{\mathrm{A}}$ \\
HR $(\mathrm{bpm})$ & $713 \pm 8.9$ & $697 \pm 12.3$ \\
IVSTD $(\mathrm{mm})$ & $0.93 \pm 0.01$ & $1.11 \pm 0.04^{\mathrm{A}}$ \\
IVSTS $(\mathrm{mm})$ & $1.55 \pm 0.02$ & $1.73 \pm 0.04 \mathrm{~B}$ \\
PWTD $(\mathrm{mm})$ & $0.85 \pm 0.03$ & $1.00 \pm 0.03^{\mathrm{B}}$ \\
PWTS $(\mathrm{mm})$ & $1.60 \pm 0.03$ & $1.48 \pm 0.06$ \\
LVEDD $(\mathrm{mm})$ & $3.2 \pm 0.03$ & $3.5 \pm 0.09^{\mathrm{A}}$ \\
LVESD $(\mathrm{mm})$ & $1.48 \pm 0.03$ & $2.0 \pm 0.07^{\mathrm{A}}$ \\
FS $\%$ & $51.7 \pm 0.77$ & $41.2 \pm 1.16^{\mathrm{A}}$ \\
\hline
\end{tabular}

Transthoracic echocardiography on unanesthetized mice. HR, heart rate. Left ventricular mass index was calculated as (external left ventricular diameter in diastole ${ }^{3}-$ LVEDD $\left.^{3}\right) \times 1.055$. Fractional shortening (FS) was calculated as (LVEDD - LVESD) / LVEDD. ${ }^{A} P<0.0001$; ${ }^{B} P<0.001$, compared with control. Data are mean \pm SEM. $n=10 /$ group.

racycline to delay transgene expression was unnecessary. Multiple founder TRE-miR-208a Tg lines were established. Primary analyses indicated that miR-208a was overexpressed at similar levels; therefore we combined results from the studies of different $\mathrm{Tg}$ lines. Throughout our studies, we compared heterozygous mice carrying the $\alpha M H C-t T A$ and TRE-miR-208a transgenes (referred to hereafter as "miR-208a Tg mice") with mice heterozygous for aMHC-tTA (referred to hereafter as "control mice").

Northern blot analysis showed miR-208a levels were approximately 4-fold higher in miR-208a Tg hearts compared with control hearts (Figure 2A). In situ hybridization using a DIGlabeled miR-208a probe confirmed that miR-208a was uniformly overexpressed in most cardiomyocytes (Supplemental Figure 1; supplemental material available online with this article; doi:10.1172/JCI36154DS1). The gross heart morphology of 4-month-old miR-208a $\mathrm{Tg}$ hearts was dramatically larger relative to control littermates (Figure 2B). Accordingly, the heart weight to body weight ratios of miR-208a $\mathrm{Tg}$ animals were significantly higher than in control animals (Figure 2C). Histological sectioning and H\&E staining revealed the appearance of enlarged chambers and thickened ventricular walls in the miR-208a Tg hearts, suggestive of hypertrophic growth (Figure 2D and Supplemental Figure 2). Analysis of desmin, an intermediate filament found near the sarcomeric $Z$ line, revealed no changes in the integrity in the sarcomeric structure of miR-208a Tg cardiomyocytes (Figure 2E). Quantitative measurement of miR-208a $\mathrm{Tg}$ cardiomyocytes revealed a $52 \%$ increase in cell size relative to controls (Figure 2F). The distribution of the cell size measurements from control and $\mathrm{Tg}$ cardiomyocytes clustered around distinct peaks, indicating that the hypertrophy in miR-208a Tg heart is fairly uniform (Figure $2 \mathrm{G})$. Together, these results indicate that miR-208a overexpression in the mouse heart induced hypertrophic growth.

Cardiac hypertrophy is the heart's primary response to stress caused by pathological and physiological hemodynamic overload, abnormal hormonal signaling, and certain inherited disorders involving particular transcription factors and contractile proteins (19). Hypertrophic growth involves enhanced protein synthesis, increased sarcomeric density, and increased cardiomyocyte size that culminates into structural remodeling of the heart. Although cardiac hypertrophy is considered an adaptive mechanism to sustain cardiac output, prolonged pathological hypertrophy has adverse consequences associated with heart failure and sudden death (24). Analysis of cardiac function by echocardiography on 3-month-old animals revealed that miR-208a $\mathrm{Tg}$ hearts displayed thickening of the ventricular walls (anterior wall in diastole and systole, posterior wall in diastole), an increase in left ventricular diameter (left ventricular diameter in diastole and systole), and deterioration in cardiac function, as indicated by decreased fractional shortening (Figure $2 \mathrm{H}$ and Table 1). We also measured cardiac function in 7-month-old animals and obtained similar results (Supplemental Table 1).

A molecular hallmark of cardiac hypertrophy is the upregulation of $\beta \mathrm{MHC}$ and the cardiac hormone atrial natriuretic factor (ANF) in the adult heart $(19,20)$. Consistent with hypertrophic growth, we observed increased expression of $\beta \mathrm{MHC}$ transcripts and proteins, by real-time PCR and Western blot analyses, respectively, in miR-208a Tg hearts (Figure 3, A and B). Unexpectedly, no significant changes in ANF transcript levels were detected (Figure 3A).

Changes in the expression levels of specific miRNAs have been reported in diseased human hearts and in animal models of heart disease, pointing to their potential roles in cardiomyopathies $(15,16,25-27)$. Since $\beta M H C$ expression is a hallmark of cardiac hypertrophy and because $\beta$ MHC and miR-208b appear to be co-regulated, we surmised that miR-208b expression would also increase during cardiac hypertrophy. Using a mouse model of cardiac hypertrophy in which the aorta was surgically constricted to produce chronic pressure overload, we indeed found miR-208b expression induced during hypertrophic growth (Figure 3C). Furthermore, miR-208b expression was found induced in adult miR-208a Tg hearts (Figure 3D). We also analyzed the expression of miRNAs whose expression levels have been reported altered in cardiac hypertrophy. While previous studies reported decreased miR-1, miR-133, and miR-29a expression levels and increased miR-125b expression levels in cardiac hypertrophy $(15,16,25-27)$, in our study the expression levels of those miRNAs were not significantly affected in miR-208a $\mathrm{Tg}$ hearts relative to control hearts (Figure 3D). Together, those data demonstrate that miR-208a induced hypertrophic growth without affecting all aspects of the hypertrophic growth response pathway.

We also determined whether the effects of miR-208a overexpression on hypertrophy could be recapitulated in vitro using isolated rat neonatal cardiomyocytes. Cardiomyocytes were transduced with miR-208a-expressing or control adenoviruses, then immunostained for $\alpha$-actinin or $\beta \mathrm{MHC}$ (Figure 3E). Consistent with the role of miR-208a in the induction of cardiac hypertrophy in vivo, overexpression of miR-208a in isolated cardiomyocytes increased cell size and $\beta \mathrm{MHC}$ expression but did not significantly affect ANF levels (Figure 3, E-H). Conversely, knockdown of miR-208a by introducing chemically modified oligonucleotides (2'O-methyl modified) into isolated cardiomyocytes resulted in decreased $\beta M H C$ expression, but the size of cardiomyocytes and ANF expression were not affected (Figure 3, E-H). Taken together, these results using in vivo and in vitro strategies suggest that miR-208a influences a subset of genes important in cell growth rather than activating a broader hypertrophic pathway.

Spatial distribution of $\beta M H C$ in miR-208 a Tg hearts is focal. Increased $\beta \mathrm{MHC}$ expression during cardiac hypertrophy is a well-estab- 
A

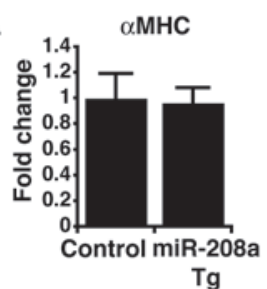

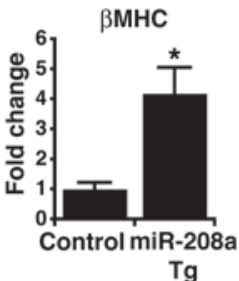

$\mathrm{Tg}$

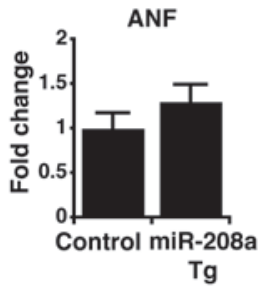

B

Control miR-208a Tg

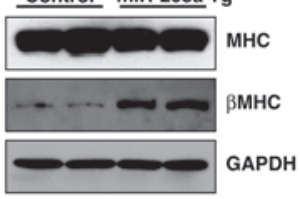

$\mathrm{Tg}$

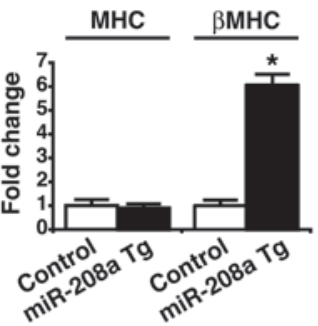

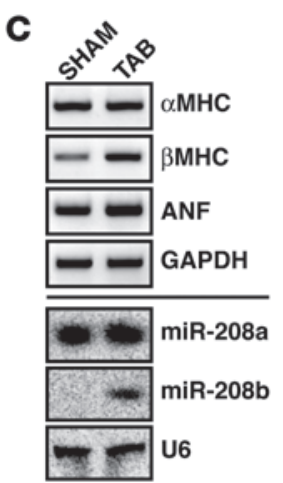

E

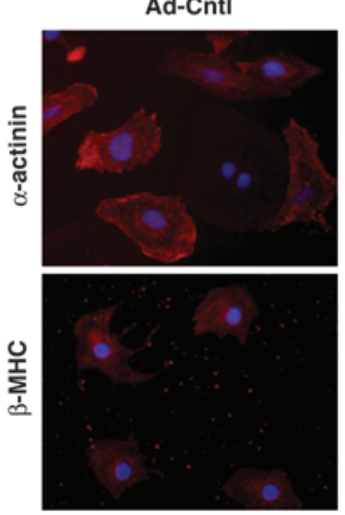

$\mathbf{F}$

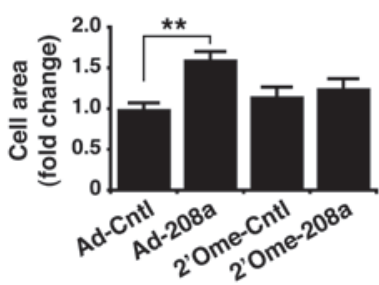

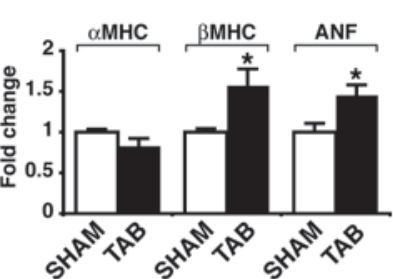
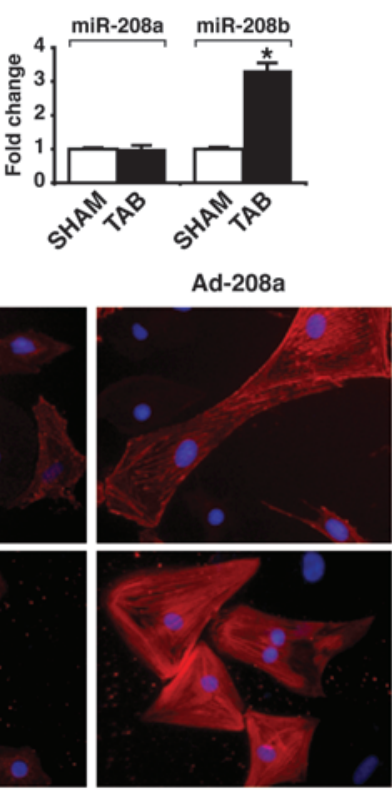

G

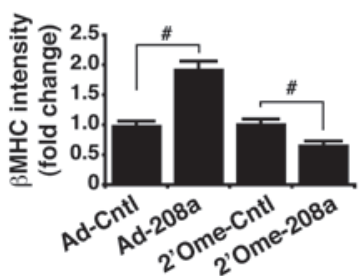

D Control miR-208a Tg
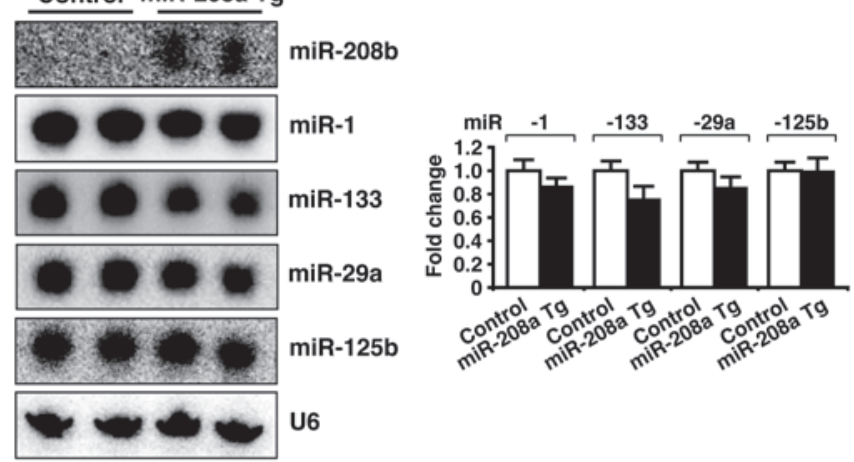

2'Ome-Cntl
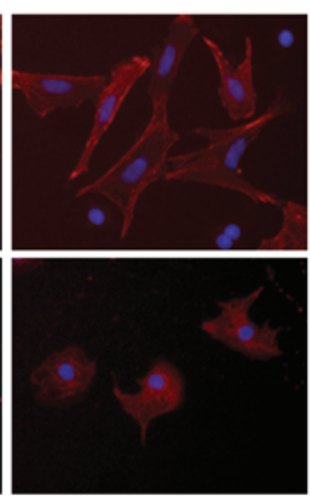

H

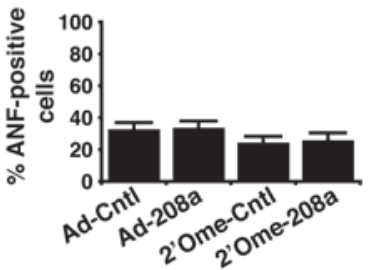

Figure 3

miR-208a overexpression induces hypertrophic gene expression. (A) Transcripts for $\alpha M H C, \beta M H C$, and ANF were detected by real-time PCR in 4-month-old hearts from control and miR-208a Tg mice $(n=5$ per genotype). Data are the mean fold change in expression \pm SEM. ${ }^{*} P<$ 0.01. (B) Left: Western blot analysis of total $\mathrm{MHC}$ and $\beta M H C$ protein levels in adult control and miR-208a Tg hearts. Right: Quantitative analysis of fold change in protein levels. ${ }^{\star} P<0.01$. (C) Top left: RT-PCR was used to detect $\alpha M H C, \beta M H C$, and ANF transcripts in wild-type hearts following 3 weeks thoracic aortic banding (TAB) or in surgical sham hearts, which were used as controls. Bottom left: miR-208a and miR-208b were detected by Northern blot analysis. U6 served as a loading control. Right: Quantitative analysis of fold change in expression of mRNAs and miRNAs. ${ }^{*} P<0.01$. (D) Northern blot and quantitative analysis of expression of miRNAs using hearts from control and miR-208a Tg mice. (E-H) Isolated rat neonatal cardiomyocytes were transduced with miR-208a and control adenoviruses (Ad-208 and Ad-Cntl, respectively) or transfected with oligonucleotides antisense to miR-208a or control oligonucleotides (2'Ome-208a and 2'Ome-Cntl, respectively). (E) Cardiomyocytes were stained for $\alpha$-actinin or $\beta M H C$ proteins. Original magnification, $\times 200$. (F) Fold change in mean cell area \pm SEM of $\alpha$-actinin-immunostained cardiomyocytes were treated with adenoviruses or oligonucleotides $\left(n=100\right.$ cells/condition; $\left.{ }^{* *} P<4 \times 10^{-12}\right)$. (G) Fold change in mean fluorescent intensity \pm SEM of $\beta M H C$-immunostained cardiomyocytes treated with adenoviruses or oligonucleotides $(n=100$ cells/condition; $\left.{ }^{\sharp} P<3 \times 10^{-7}\right)$. (H) Cardiomyocytes were treated with adenoviruses or antisense 2' $\mathrm{O}$-methyl oligonucleotides and were scored for ANF staining ( $n \approx 425$ cells/condition). 

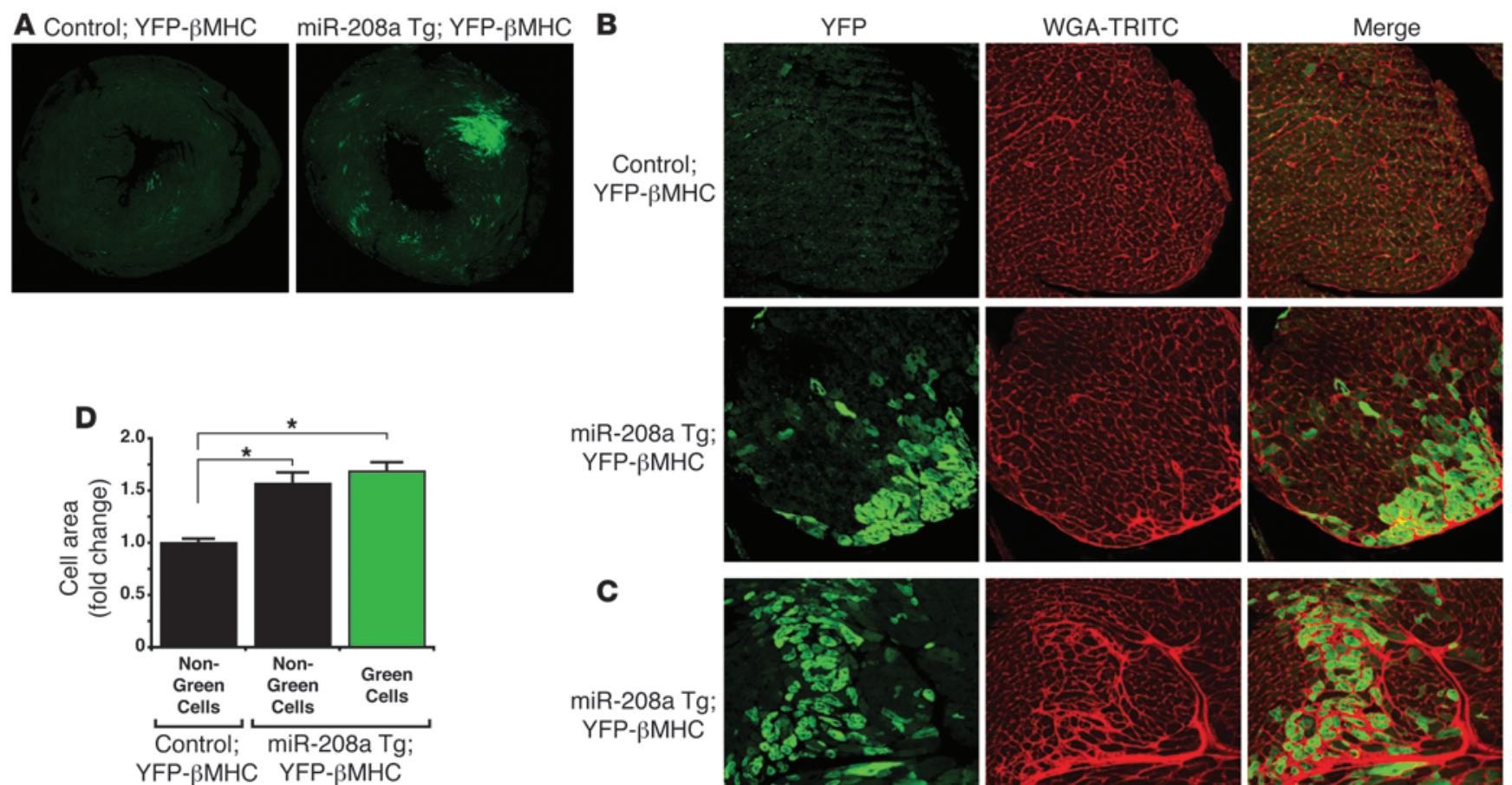

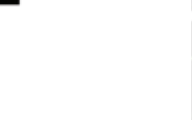
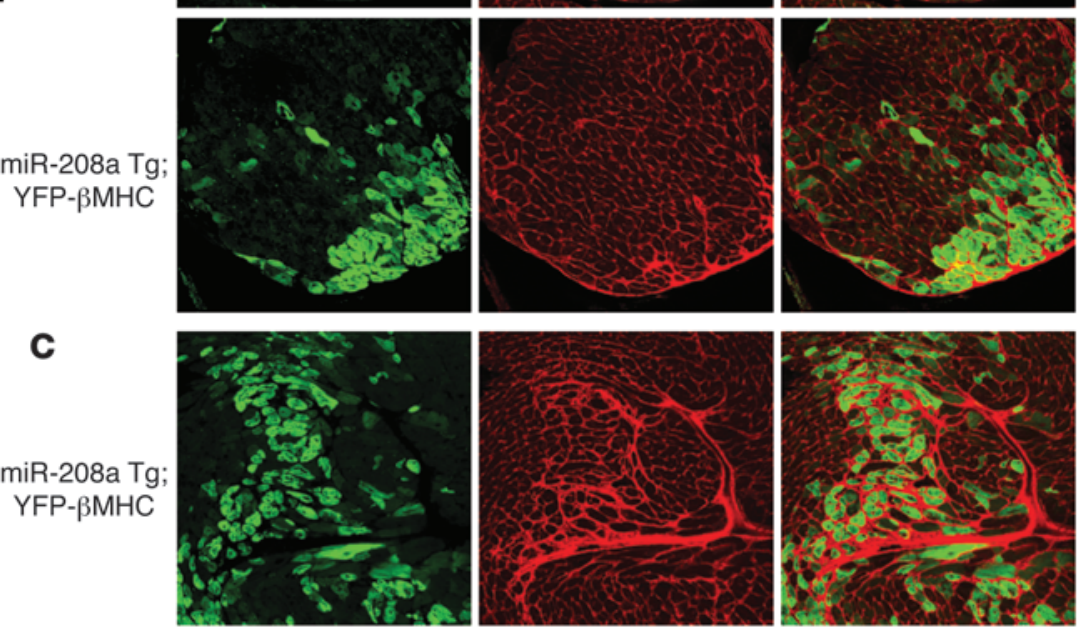

\section{Figure 4}

Distribution of YFP-BMHC fusion protein in miR-208a Tg hearts. (A) Confocal fluorescent images of coronal sections from control and miR-208a Tg hearts. Overlapping images were stitched together using ImageJ. Original magnification, $\times 40$. (B) Papillary muscle from control and miR-208a Tg hearts imaged for YFP- $\beta$ MHC (green) expression and wheat germ agglutinin-TRITC staining (red). Original magnification, $\times 200$. (C) Representative fluorescent images of YFP- $\beta M H C$ expression (green) in an area of interstitial fibrosis (red) in miR-208a Tg hearts. Original magnification, $\times 200$. (D) Mean cell area \pm SEM of cardiomyocytes from miR-208a Tg; YFP- $\beta M H C$ and control; YFP- $\beta$ MHC hearts. Cells measured for area were also scored for presence or absence of YFP- $\beta$ MHC expression $\left(n=100 /\right.$ genotype; $\left.{ }^{\star} P<0.001\right)$.

lished phenomenon and is thought to contribute to the overall poor functioning of the hypertrophic heart (28-30). To better assess the effects of miR-208a on the expression of $\beta \mathrm{MHC}$, we employed a mouse strain harboring a $\beta \mathrm{MHC}$ indicator allele, in which the yellow fluorescent protein (YFP) sequence is fused to the Myb7 gene (31). We bred this allele into the miR-208a Tg line. The YFP- $\beta$ MHC was highly expressed in neonatal cardiomyocytes, but was essentially unexpressed in adult hearts (ref. 31, Figure $4 \mathrm{~A}$, and data not shown). We observed dramatically increased YFP- $\beta$ MHC protein levels in the miR-208a Tg hearts (Figure 4A and Supplemental Figure 3). However, YFP- $\beta$ MHC expression did not increase in all cardiomyocytes of miR-208a Tg hearts. Rather it was intensely upregulated only in areas associated with interstitial fibrosis (Figure 4, B and C, and Supplemental Figure $3)$. This observation is consistent with an earlier report in which the distribution of $\beta \mathrm{MHC}$ was analyzed and found correlated with fibrosis in an established mouse model of cardiac hypertrophy (31). Inhibition of thyroid hormone synthesis by propylthiouracil (PTU) results in hypothyroidism and increases $\beta \mathrm{MHC}$ expression due to loss of thyroid hormone-mediated repression at the $\beta$ MHC promoter (22). PTU treatment of adult miR-208a $\mathrm{Tg}$ mice resulted in uniform upregulation of YFP- $\beta$ MHC expression throughout the myocardium after 4 weeks, indicating that expression of the $\beta \mathrm{MHC}$ indicator allele recapitulates $\beta \mathrm{MHC}$ expression (Supplemental Figure 4). Thus, even though in situ hybridization analyses for mature miR-208a revealed uniform overexpression in miR-208a Tg cardiomyocytes (Supplemental Figure 1), $\beta \mathrm{MHC}$ re-expression occurred only in a subset of cardiomyocytes associated with fibrosis.

We next tested whether $\beta$ MHC re-expression correlated with the miR-208a-induced hypertrophic growth of individual cardiomyocytes. To do this, we compared the cell areas of miR-208a Tg;YFP$\beta \mathrm{MHC}$ cardiomyocytes with the areas of control YFP- $\beta \mathrm{MHC}$ cardiomyocytes lacking the miR-208a transgene. No association between the state of YFP- $\beta$ MHC expression and cell area increase was observed (Figure 4D). Thus, cardiomyocytes from miR-208a $\mathrm{Tg}$ hearts were significantly larger than the cardiomyocytes from control hearts independently of whether they were positive or negative for YFP- $\beta$ MHC expression (Figure 4D). Taken together, these observations demonstrate that $\beta \mathrm{MHC}$ expression is not an obligate component of miR-208a-induced hypertrophic growth and that overexpression of miR-208a alone is sufficient to induce hypertrophic growth in cardiomyocytes even when they show no re-activation of $M y b 7$ gene expression.

Targeted deletion of miR-208a alters cardiac gene expression. Having demonstrated that miR-208a is sufficient for hypertrophic growth, we sought to examine miR-208a function using a loss-of-function approach in miR-208a knockout mice. We replaced the genomic sequence encoding miR-208a by homologous recombination with a neomycin selection cassette flanked by loxP sites (Figure 5, A and B). The selection cassette was subsequently excised by Cre-mediated recombination, leaving only a small footprint of exogenous 
A

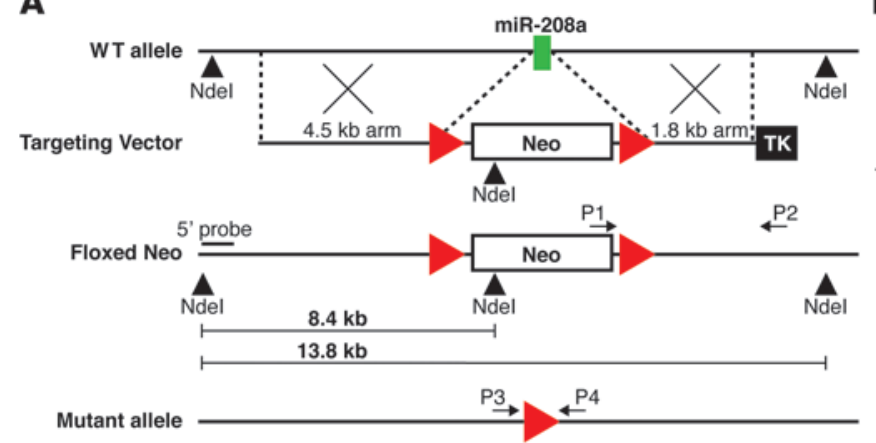

B

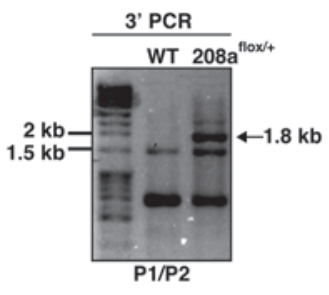

C
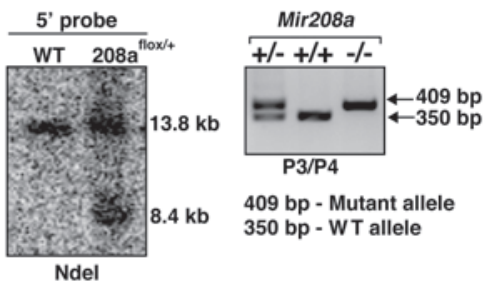

409 bp - Mutant allele $350 \mathrm{bp}-$ WT allele
D

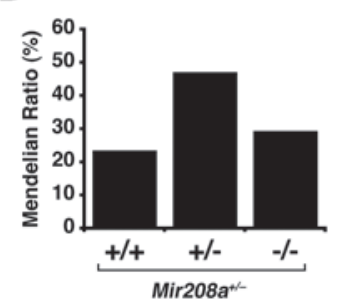

E Mirzosa

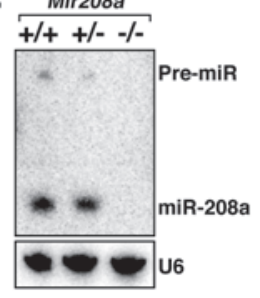

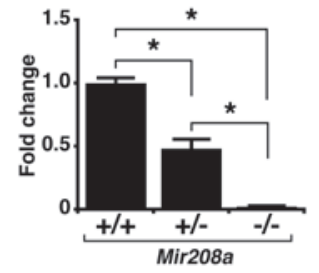

H
F

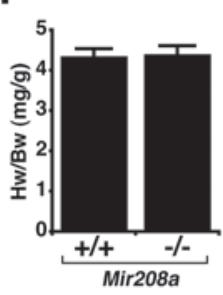

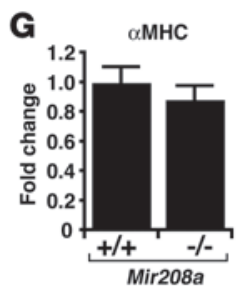
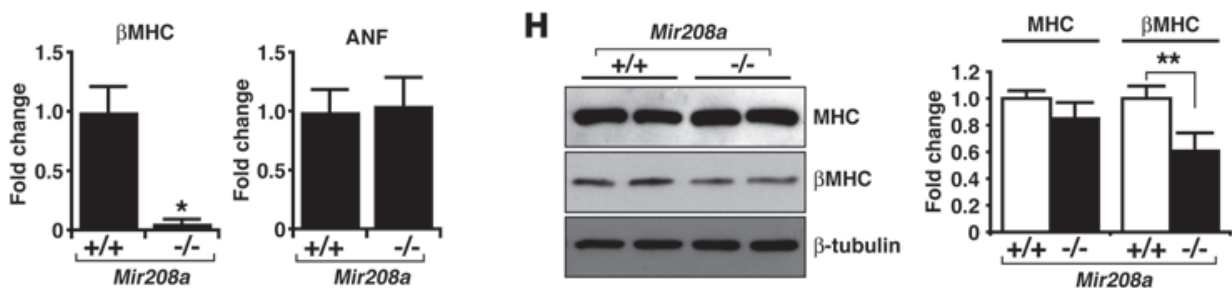

I
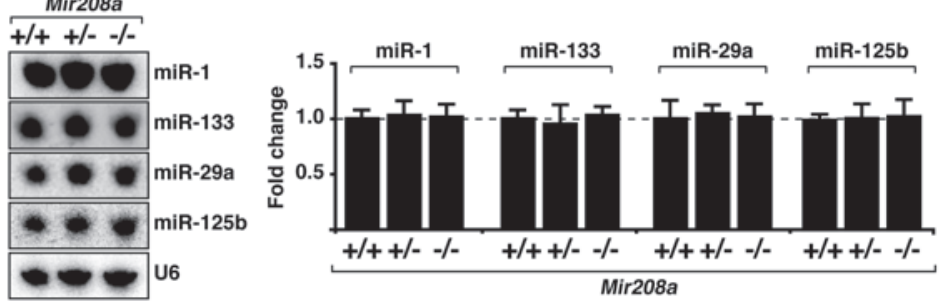

Figure 5

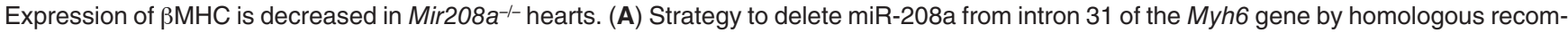
bination. The miR-208a coding sequence (green box) was replaced by a neomycin selection cassette flanked by loxP sites (red triangles). The selection cassette was excised from the germline by mating with mice that ubiquitously expressed Cre recombinase, thus creating a mutant allele that contained a single loxP sequence in place of miR-208a. Genotyping PCR primers and 5' probes are indicated. Ndel, restriction enzyme site; P1, primer binding site 1. (B) The occurrence of the intended recombination event in mouse ES cells was confirmed by PCR and Southern blot analyses. (C) The increased length of the mutant allele was the basis for a PCR-based genotyping strategy. (D) Genotypes of progeny from mating miR-208a mice were born at a Mendelian ratio $(n=128)$. (E) Northern blot analysis for miR-208a expression in hearts from wild-type

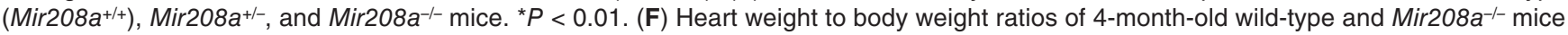
( $n=25 /$ genotype). (G) Transcripts for $\alpha$ MHC, $\beta$ MHC, and ANF were detected by real-time PCR in hearts from wild-type and Mir208a ${ }^{-/-}$mice $\left(n=5 /\right.$ genotype). Values are presented as the fold change in mean expression \pm SEM. ${ }^{*} P<0.01$. (H) Western blot analysis of total MHC and $\beta \mathrm{MHC}$ protein levels in hearts from wild-type and Mir208a $a^{-/}$mice. ${ }^{* *} P<0.05$. (I) Northern blot analysis of miRNA expression using hearts from wild-type, Mir208a ${ }^{+-}$, and Mir208a $a^{-/-}$mice.

DNA in place of miR-208a (Figure 5C). Since miR-208a is located within an intron of the Myb6 gene, we confirmed that the splicing pattern of the $\alpha \mathrm{MHC}$ transcript was unaffected by the mutant miR-208a allele (Supplemental Figure 5).

The progeny that resulted from mating Mir208a $a^{+/-}$mice were viable and born at an expected Mendelian ratio (Figure 5D). miR-208a expression was halved in Mir208a $a^{+/}$hearts compared with the wildtype hearts and was undetectable in Mir208a-/- hearts (Figure 5E and Supplemental Figure 1). Hearts of 12- to 16-week-old Mir208a-/mice did not display any gross morphological abnormalities and appeared normal compared with wild-type littermates (data not shown). Furthermore, no differences in heart weight to body

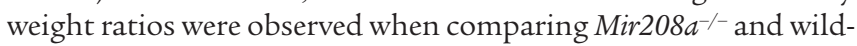
type mice (Figure $5 \mathrm{~F}$ ). Those results are consistent with a recent report in which miR-208a was shown to not be required for normal heart development and function (17). 
A

B
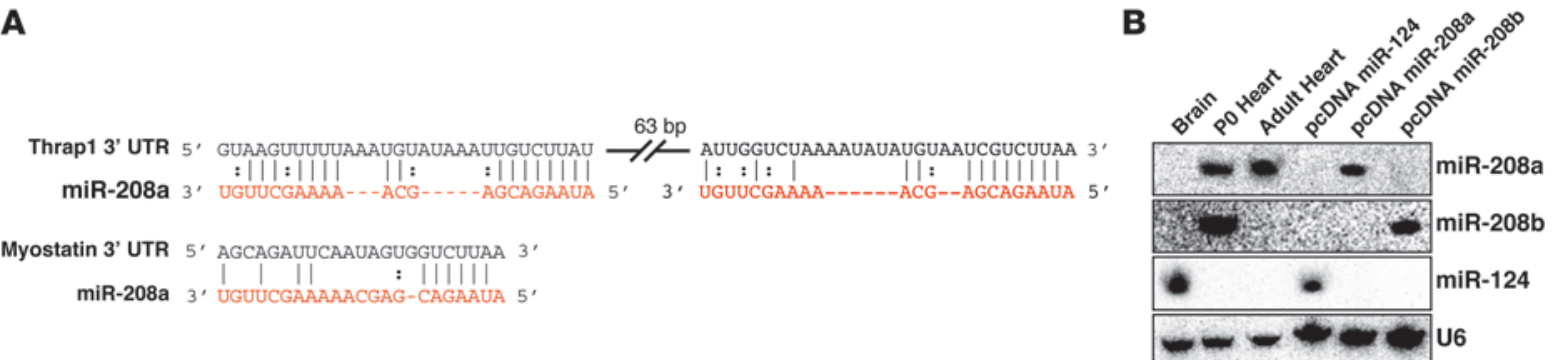

C

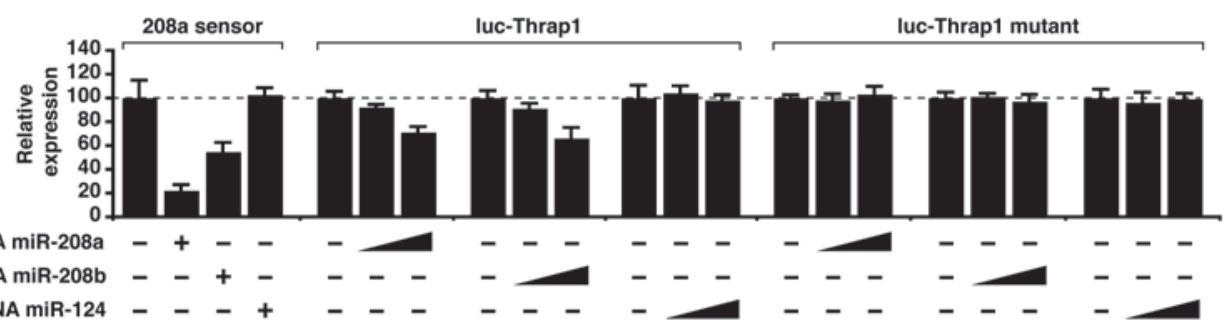

D

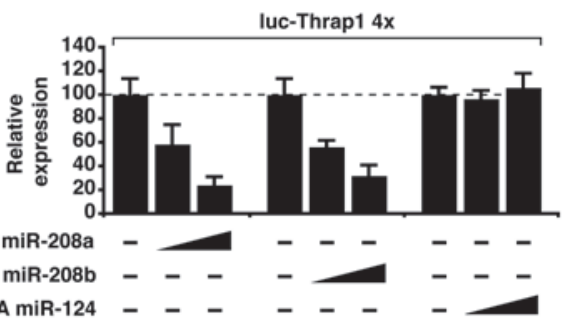

$\mathbf{F}$

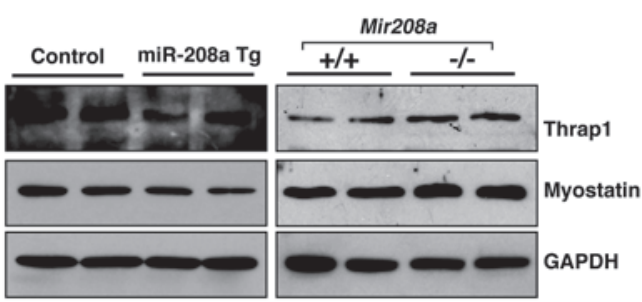

E
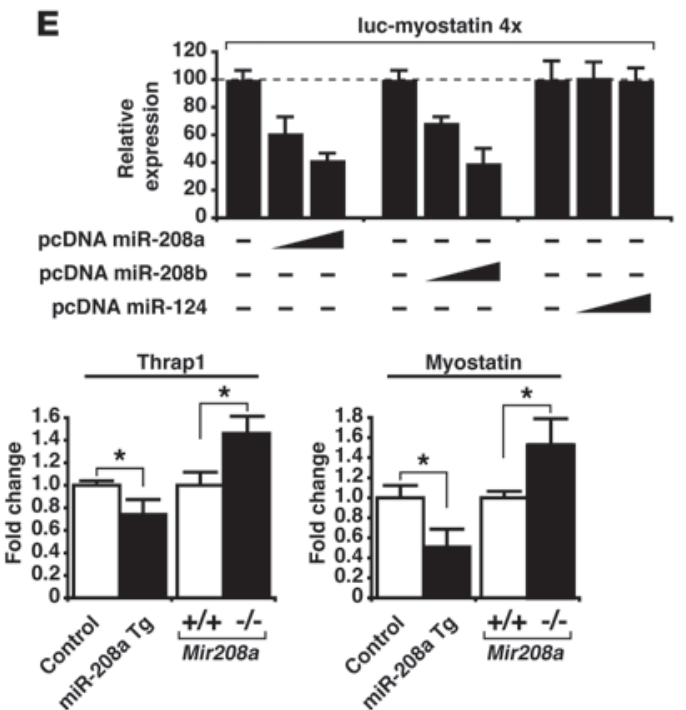

\section{Figure 6}

miR-208a and miR-208b repress the expression of Thrap1 and myostatin. (A) Sequence alignment between miR-208a and candidate binding sites in the 3' UTR of Thrap1 and myostatin. (B) Northern blot analysis demonstrated that miR-208a, miR-208b, and miR-124 expression plasmids produced mature miRNAs when transfected into 293T cells. Total RNA from mouse brain and neonatal and adult hearts was included as a control. U6 served as a loading control. (C) 293T cells were transfected with a luciferase reporter designed to detect miR-208a expression (208a sensor), along with the indicated miRNA expression plasmids. A Thrap1 3' UTR (luc-Thrap1) and a mutated Thrap1 3' UTR (luc-Thrap1 mutant) were also tested. Values are mean luciferase activity \pm SD relative to the luciferase activity of reporters cotransfected with an empty expression plasmid. The dotted line indicates the basal level of luciferase activity in control (i.e., the luciferase vector alone). (D) A luciferase reporter with duplicated Thrap1 binding sites (luc-Thrap14x) was cotransfected with miRNA expression plasmids and luciferase activity determined. (E) A luciferase reporter with 4 repeats of the putative myostatin binding site was cotransfected with miRNA expression plasmids and luciferase activity determined. (F) Western blot analysis for Thrap1 and myostatin protein levels in hearts from 4-month-old miR-208a Tg versus

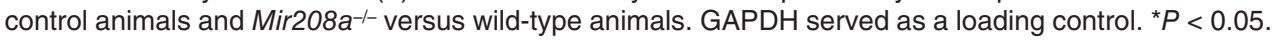

Consistent with the role of miR-208a in the regulation of cardiac hypertrophic growth and $\beta \mathrm{MHC}$ expression, we found that $\beta \mathrm{MHC}$ transcript and protein levels were significantly reduced, while $\alpha \mathrm{MHC}$ and ANF transcript levels were unchanged in Mir208a-/hearts (Figure 5, G and $\mathrm{H}$ ). This result complements the elevation of $\beta$ MHC transcript and protein levels observed in miR-208a Tg hearts (Figure 3, A and B). Together, those genetic data provide convincing evidence that miR-208a is important for the regulation of $\beta \mathrm{MHC}$ expression. We also examined the expression of miRNAs that were downregulated in miR-208a Tg hearts (Figure 3D). Surprisingly, we found that the expression of those miRNAs appeared unchanged in Mir208a $\mathrm{a}^{-/-}$hearts, indicating that their expression is not dependent upon miR-208a (Figure 5I).

$m i R-208$ and $m i R-208 b$ repress the expression of thyroid hormone-associated protein 1 and myostatin. Utilizing the web-based TargetscanHuman database (http://www.targetscan.org/), we selected several predicted miR-208a target genes for experimental scrutiny $(32,33)$. A target site located in the $3^{\prime}$ UTR of thyroid hormone-associated pro- 
Table 2

Summary of 4-month surface ECG findings

\begin{tabular}{|c|c|c|c|c|c|}
\hline Mouse & HR (bpm) & PR (ms) & QRS (ms) & QT (ms) & QTc (ms) \\
\hline Control $(n=11)$ & $500 \pm 24$ & $38 \pm 1$ & $9 \pm 0$ & $48 \pm 2$ & $43 \pm 1$ \\
\hline miR-208a $\operatorname{Tg}(n=16)$ & $460 \pm 17$ & $46 \pm 2^{A}$ & $9 \pm 1$ & $50 \pm 2$ & $43 \pm 1$ \\
\hline Mir208a $a^{++}(n=8)$ & $491 \pm 22$ & $37 \pm 1$ & $9 \pm 0$ & $53 \pm 1$ & $48 \pm 1$ \\
\hline Mir208a $a^{-1-}(n=10)$ & $503 \pm 22$ & $49 \pm 1^{B}$ & $11 \pm 1$ & $50 \pm 2$ & $54 \pm 2$ \\
\hline
\end{tabular}

${ }^{A} P<0.01 ;{ }^{B} P<0.001$, compared with control.

tein 1 (Thrap1) is among the most heavily weighted target sites for miR-208a and was chosen for study since thyroid hormone signaling is a known repressor of $\beta$ MHC transcription $(34,35)$. Upon closer inspection of the Thrap 1 3' UTR, we identified a second conserved miR-208a target site located approximately $60 \mathrm{bp}$ downstream of the first target site (Figure 6A and Supplemental Figure 6).

In addition, myostatin is also predicted to be a miR-208a regulatory target, with a single miR-208a target site in its 3' UTR (Figure 6A and Supplemental Figure 6). Myostatin stood out as an interesting candidate, given its role as a repressor of hypertrophic growth in skeletal muscle $(36,37)$. Myostatin is also expressed in cardiac muscle, although to a much lesser degree than it is found in skeletal muscle, and genetic inactivation of myostatin in mice has been recently linked to cardiac hypertrophy (38-41).

As a first step toward determining which genes are targeted by miR-208a and miR-208b, we cloned genomic fragments encoding miR-208a, miR-208b, and miR-124 into plasmids for overexpression in cultured cells (Figure 6B). We hypothesized that similar sequences and identical seed regions shared by miR-208a and miR-208b would enable them to repress similar sets of genes, while miR-124 is a brainspecific miRNA and served as a control miRNA for specificity.

Thrap1 is part of the thyroid hormone nuclear receptor complex and can positively and negatively influence transcription $(42,43)$. Thus we reasoned that repression of Thrap 1 by miR-208a might account for the increased $\beta$ MHC expression in miR-208a Tg hearts (Figure 3, A and B). In agreement with this notion, cotransfection of a luciferase gene with the Thrap1 3' UTR cloned immediately downstream (luc-Thrap1) and the miR-208a expression plasmid in cultured cells resulted in repressed luciferase activity (Figure 6C). Expression of miR-124 with luc-Thrap1 had no such effect upon luciferase activity, which indicated that miR-208a repression of luc-Thrap1 was specific. To further confirm this specificity, we mutated the candidate miR-208a target sites (luc-Thrap1 mutant), which resulted in the complete loss of miR-208a-mediated repression (Figure 6C and Supplemental Figure 6). As we predicted from the sequence similarity shared by miR-208a and miR-208b, we found that miR-208b also repressed the luc-Thrap1 luciferase activity (Figure 6C). As another demonstration of miR-208a and miR-208b targeting of Thrap1 and to test whether increasing the number of target sites would also increase the degree of repression, we duplicated the Thrap1 target sites downstream of the luciferase gene (luc-Thrap1 4x). Indeed, increasing the target site number resulted in a pronounced decrease in luciferase activity, indicating that target site number is an important factor in miRNA-mediated repression (Figure 6D).

In order to directly test whether miR-208a could repress the expression of myostatin, we constructed 4 repeats of the myostatin target sequence downstream of a luciferase gene (luc-myostatin $4 \times$ ) and cotransfected with miRNA expression plasmids. Transfection of either miR-208a or miR-208b plasmids repressed luc-myostatin $4 \times$ luciferase activity (Figure 6E). Transfection of the miR-124 plasmid caused no decrease in luc-myostatin $4 \times$ luciferase activity and confirmed that miR-208a and miR-208b specifically target the myostatin 3' UTR (Figure 6E).

To determine whether Thrap 1 and myostatin were regulated in vivo by miR-208a, we tested whether their expression was altered in our miR-208a gain- and loss-of-function mouse models. The transcript levels of Thrap1 and myostatin appeared unchanged in the miR-208a Tg and Mir208a-/- hearts (Supplemental Figure 6). However, the protein levels of Thrap1 and myostatin were repressed in miR-208a Tg hearts compared with control hearts (Figure 6F). Conversely, the protein levels of Thrap1 and myostatin were elevated in Mir208a-/- hearts compared with hearts from wild-type littermates (Figure 6F). Taken together, these observations demonstrated that Thrap 1 and myostatin are bona fide miR-208a targets.

miR-208a is a regulator of the cardiac conduction system. To determine whether miR-208a overexpression disturbed cardiac conduction, we recorded surface ECGs of 4-month-old miR-208a Tg and control mice. Analysis of the ECG recordings showed significantly prolonged PR intervals in miR-208a Tg mice compared with control mice (Table 2 and Figure 7A). The PR interval is the period of time between the onset of atrial depolarization and the onset of ventricular depolarization; abnormal prolongation of the PR interval is clinically considered first-degree AV block. No significant differences were detected in other ECG parameters, such as QRS, QT, or QTc intervals (Table 2). We also recorded and analyzed surface ECGs from 1- and 6-month-old animals and obtained similar results (Supplemental Table 2). Interestingly, approximately $30 \%$ of the miR-208a Tg mice also had second-degree AV blocks, in which one or more of the electrical impulses from the atria failed to pass through the $\mathrm{AV}$ node to the ventricles, causing failures in ventricular contraction (Figure 7B). Second-degree AV blocks appeared on the ECG tracings as P waves (atrial depolarizations) without subsequent occurrence of QRS complexes (ventricular depolarizations) (Figure 7B). Taken together, the development of progressive heart blocks in miR-208a Tg mice demonstrates that miR-208a overexpression results in cardiac conduction abnormalities and suggests that miR-208a regulates cardiac conduction system components.

We and others have found that genetic deletion of miR-208a does not affect viability or cause gross morphological heart defects but is required for stress-dependent heart growth (Figure 5 and ref. 17). To determine whether miR-208a is also required for proper heart electrophysiology during normal conditions, we monitored heart

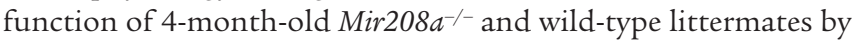
surface ECGs and found that miR-208a is necessary for proper cardiac conduction. The ECGs of approximately $80 \%$ of the Mir $208 \mathrm{a}^{-/-}$ mice lacked $\mathrm{P}$ waves preceding QRS complexes, suggesting that Mir208a $a^{-/}$mice suffer atrial fibrillation (Figure 7, C and D). Consistent with a defect in atrial conduction, the PR intervals in Mir208 $a^{-/-}$mice were significantly prolonged compared with wildtype animals (Table 2). Collectively, the ECG analyses of miR-208a $\mathrm{Tg}$ and Mir208a $\mathrm{a}^{-/-}$mice demonstrate that miR-208a is an important component of the cardiac conduction system. 
A

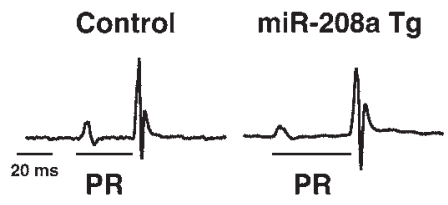

C

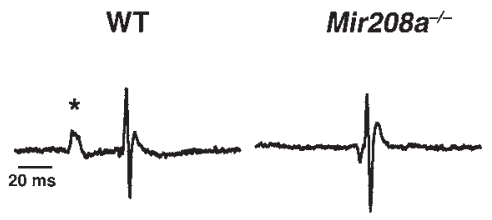

B

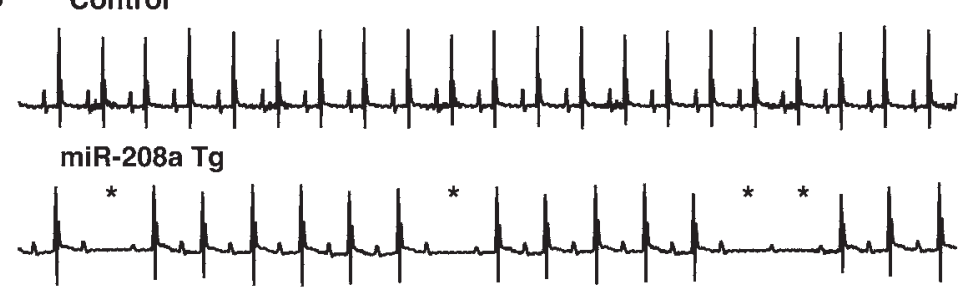

D WT

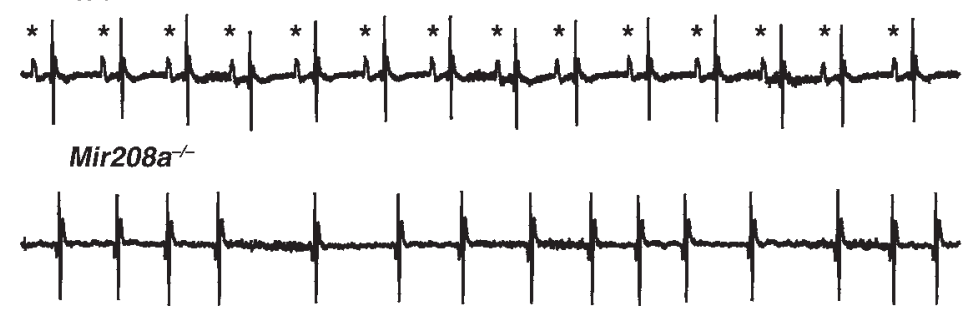

Figure 7

$\mathrm{miR}-208 \mathrm{a}$ is sufficient to induce arrhythmias and is required for proper cardiac conduction. (A) Representative waveforms in lead I indicate the location and relative duration of PR intervals in 4-month-old miR-208a Tg and control mice. (B) Representative ECGs in lead I of 4-month-old miR-208a Tg and control mice. Asterisks mark missing QRS complexes and indicate occurrences of second-degree atrioventricular block. (C) Representative waveforms in lead I from Mir208a $a^{-1-}$ and wild-type mice. Asterisk indicates the normal position of the $P$ wave, which was absent in Mir208a ${ }^{-/-}$mice. (D) Representative ECGs in lead I from 4-month-old Mir208a ${ }^{-/}$and wild-type mice. Asterisks mark the presence of the P wave.

Normal conduction is mediated by the orderly propagation of electrical impulses from one cardiomyocyte to the next. The connexin proteins are gap junction proteins required for this propagation, and their altered expression is a common feature in a variety of chronic human heart diseases associated with increased risk of arrhythmias and sudden death (44-47). Studies of mouse models have demonstrated that deficiencies in either connexin 43 $(\mathrm{Cx} 43)$ or $\mathrm{Cx} 40$ result in cardiac conduction defects (48). $\mathrm{Cx} 43$ is expressed in cardiomyocytes throughout the heart, whereas $\mathrm{Cx} 40$ expression is restricted to the atria and the specialized cardiomyocytes that constitute the His bundle and Purkinje fibers (48). Previous studies suggested that abnormal connexin protein expression might account for, at least in part, the cardiac conduction defects induced by altered miR-208a levels (49-51).

We therefore evaluated the expression of $\mathrm{Cx} 43$ and $\mathrm{Cx} 40$ in hearts from 4-month-old miR-208a Tg and Mir208a $a^{-/-}$mice. Transcript analysis by real-time PCR did not reveal any readily apparent changes to Cx43 transcript levels in either miR-208a Tg or Mir208a $a^{-/-}$hearts (Figure 8, A and B). Though Cx40 transcript levels were not affected in miR-208a Tg hearts (Figure 8C), they were markedly decreased in Mir2089 $a^{-1-}$ hearts compared with wild-type (Figure 8D), indicating that miR-208a is required for Cx40 expression. Furthermore, Western blot and immunohistological analyses of hearts from Mir208a-/mice showed decreased $\mathrm{Cx} 40$ protein levels compared with wild-type hearts (Figure 8E and Supplemental Figure 7). Consistent with the Mir208a $a^{-/-}$phenotype, mice lacking Cx40 suffer cardiac conduction abnormalities, including first-degree AV block (49).

miR-208a maintains expression of cardiac transcription factors. The transcription factor homeodomain-only protein (Hop) is highly expressed within the adult murine conduction system, and Hop genetic deletion results in postnatal conduction defects accompanied by a loss of Cx40 expression (52). Therefore, we speculated that the decreased

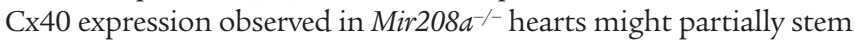
from reduced Hop expression. We evaluated Hop transcripts levels by real-time PCR using hearts from 4-month-old animals and found Hop expression to be abolished in Mir208a/- mice (Figure 8F). Accordingly, Hop protein was also undetectable in Mir208a-/- hearts (Figure 8G). Our observations indicate that Hop is not directly regulated by miR-208a, but instead that miR-208a targets a transcription factor associated with Hop and/or required for Hop expression. GATA4 and Nkx2.5 are transcriptional cofactors expressed within the cardiac conduction system of the adult heart and coordinately transactivate the expression of serum response factor-dependent promoters, including $\mathrm{Cx} 40$ and Hop $(53,54)$. Interestingly, the $3^{\prime}$ UTR of Gata 4 mRNA contained a predicted miR-208a target site (Supplemental Figure 8), and so we reasoned that the miR-208a gain- and loss-of-function phenotypes might partially result from irregular GATA4 protein expression (55). In order to directly test whether miR-208a could repress the expression of GATA4, we constructed 4 repeats of the GATA4 target sequence downstream of a luciferase gene (luc-GATA4) and cotransfected with miRNA expression plasmids. Cotransfection of miR-208a with the luc-GATA4 reporter repressed luciferase activity (Figure $8 \mathrm{H}$ ). However, cotransfection of control miR-124 caused no decrease in luciferase activity, demonstrating that miR-208a specifically targeted the $3^{\prime}$ UTR of GATA4 (Figure 8H). To determine whether GATA4 is regulated in vivo by miR-208a, we tested whether GATA4 expression was altered in the Mir208a/- hearts. Indeed, GATA4 protein levels were elevated in Mir208 ${ }^{-/}$hearts compared with hearts from wild-type littermates (Figure 8I). Consistent with posttranscriptional regulation, the GATA4 transcript levels were unchanged in Mir208a-/- hearts (data not shown). Taken together, these observations demonstrate that miR-208a directly targets the cardiac transcription factor GATA4.

\section{Discussion}

In this study, we investigated the function and molecular mechanisms of the miR-208 family in adult heart physiology. Our findings, which we believe are novel, include the following: (a) miR-208a 

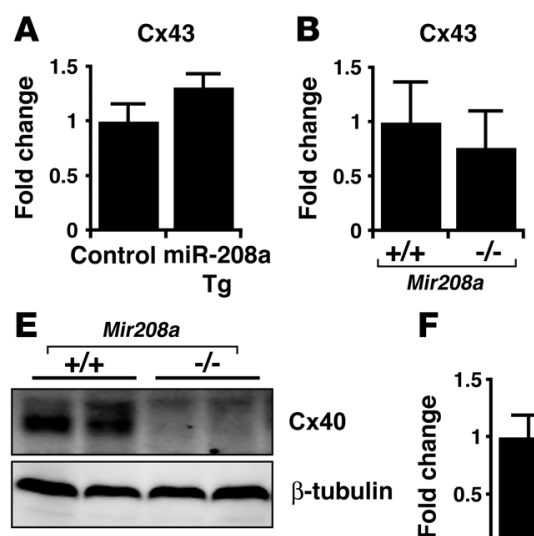

F
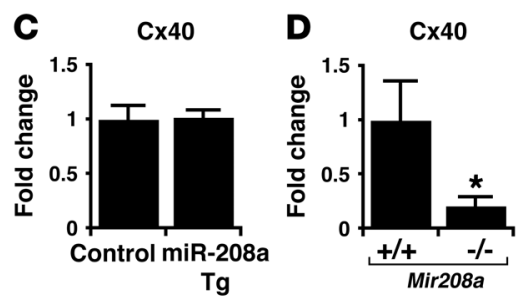

G

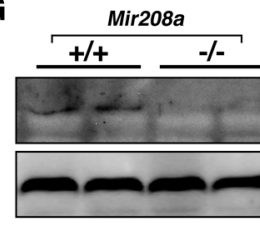

Hop
Cx40

$\beta$-tubulin

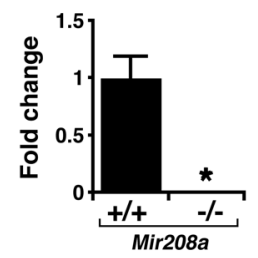

H

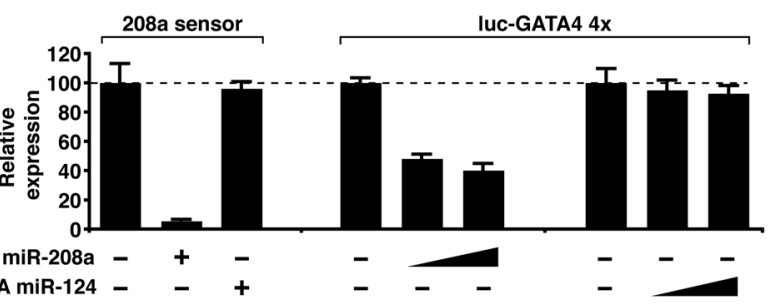

I

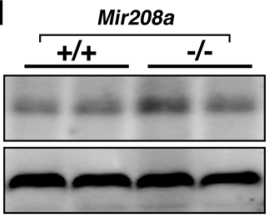

GATA4 $\beta$-tubulin

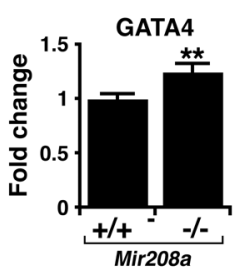

Figure 8

miR-208a is required for proper expression of gap junction protein connexin 40 and cardiac transcription factors GATA4 and Hop. (A and B) Transcripts for Cx43 were detected by real-time PCR in hearts from (A) miR-208a Tg and control mice $(n=5 / g e n o t y p e)$ and (B) wild-type and Mir208a ${ }^{-/}$mice ( $n=5 /$ genotype). (C and D) Transcripts for Cx40 were detected in hearts from (C) miR-208a Tg and control mice $(n=5$ each genotype) and (D) wild-type and Mir208a ${ }^{-/-}$mice $\left(n=5 /\right.$ genotype). Values in A-D are presented as the fold change in expression \pm SEM. ${ }^{*} P<0.01$. (E) Western blotting for Cx40 proteins using hearts from 4-month-old wild-type and Mir208 $a^{-/-}$mice. $\beta$-Tubulin served as a loading control. (F) Transcripts for Hop were detected by real-time PCR in hearts from wild-type and Mir208a ${ }^{-/}$mice $(n=5 /$ genotype). (G) Western blotting for Hop proteins using hearts from 4-month-old wild-type and Mir208a ${ }^{-/-}$mice. (H) 293T cells were transfected with a luciferase reporter designed to detect miR-208a expression and with miRNA expression plasmids, and luciferase activity was determined. A luciferase reporter with 4 repeats of the putative miR-208a binding site was also cotransfected with miRNA expression plasmids and luciferase activity determined. Values are mean luciferase activity $\pm S D$ relative to the luciferase activity of reporters cotransfected with empty expression plasmid. (I) Western blotting for GATA4 proteins using hearts from 4-month-old wild-type and Mir208a ${ }^{-/-}$mice. ${ }^{* *} P<0.05$.

and miR-208b are members of miR-208 family and are differentially expressed during heart development and heart pathology, paralleling the expression of their respective host genes, $\alpha \mathrm{MHC}$ and $\beta \mathrm{MHC}$. (b) Cardiac overexpression of miR-208a is sufficient to induce hypertrophy. (c) miR-208a-induced $\beta \mathrm{MHC}$ expression is restricted to a subset of cardiomyocytes associated with fibrosis. (d) $\beta$ MHC expression is dramatically decreased in Mir208a-/- mouse hearts. (e) Both miR-208a and miR-208b target Thrap1 and myostatin, 2 important negative regulators of muscle growth and hypertrophy. (f) miR-208a is necessary for normal cardiac conduction. miR-208a gain- and lossof-function are associated with arrhythmias. Together, the results of our study provide critical functional and mechanistic insights into how miRNAs participate in the regulatory cascade to modulate cardiac remodeling and normal physiology.

The $\beta M H C$ and $\alpha M H C$ isoforms are major contractile proteins of cardiomyocytes and differ primarily in their ability to convert ATP to mechanical work at different rates, thus their relative protein expression ratio affects contractility of the cardiac sarcomeres $(56,57)$. Increased expression of $\beta \mathrm{MHC}$ is a common feature of cardiac hypertrophy and heart failure that reduces contractile performance and is thought to be a maladaptive response (28-30). The shift towards $\beta \mathrm{MHC}$ is reversible under particular conditions that are associated with improved cardiac performance, including the regression of hypertrophy and human patients that respond favorably to $\beta$ blocker therapy (58-62). Furthermore, mutations in the Myb7 gene are commonly associated with hypertrophic cardiomyopathies (63). Therapies that inhibit the maladaptive features of hypertrophy might be useful in improving the function of the diseased heart (20). However, the molecular events that induce hypertrophy and govern hypertrophic gene expression are not well defined. More specifically, it is not fully understood how $\beta \mathrm{MHC}$ is regulated during cardiac remodeling and how its reactivation contributes to functional maladaptation. Our experimental results demonstrate that miR-208a is sufficient to induce cardiac hypertrophy in $\mathrm{Tg}$ hearts. Interestingly, the hypertrophic growth induced by miR-208a is only accompanied by increased $\beta \mathrm{MHC}$ expression in a subset of hypertrophied cardiomyocytes. It will be important to perform time course experiments in the future to determine the temporal regulation of $\beta$ MHC by miR-208a.

In contrast, deletion of miR-208a resulted in decreased $\beta \mathrm{MHC}$ expression in the adult heart, providing genetic evidence that miR$208 \mathrm{a}$ is required for $\beta \mathrm{MHC}$ expression. Our results are consistent with a prior report showing that loss of miR-208a blunted stressinduced cardiac hypertrophy and $\beta \mathrm{MHC}$ reactivation (17). Strikingly, upregulated $\beta \mathrm{MHC}$ expression in miR-208a Tg hearts is tightly associated with regional fibrosis, similar to what we previously 
found in renin-induced hypertrophy (31). The strong correlation of re-expression of $\beta \mathrm{MHC}$ and fibrosis development may explain why such reactivation of fetal genes is associated with a maladaptive phenotype. Further studies to understand how induced $\beta \mathrm{MHC}$ expression is associated with fibrosis will likely shed light on the biology of cardiac hypertrophy and heart failure.

Our findings demonstrate that miR-208a posttranscriptionally represses the expression of Thrap1, a component of the thyroid hormone nuclear receptor complex. Thyroid hormone signaling has long been an established regulator of cardiac myosin heavy chain isoform expression: a surge of circulating thyroid hormone that occurs after birth transcriptionally represses $\beta M H C$ expression while activating $\alpha \mathrm{MHC}$ expression $(34,35)$. Studies have also shown that excessive administration of thyroid hormone leads to the development of cardiac hypertrophy, but the molecular mechanism was elusive (64-67). Our findings, in which increasing the level of miR-208a in Tg hearts reduced Thrap1 levels and induced hypertrophic growth, provide a link between the action of miR-208a and thyroid hormone in cardiac hypertrophy. We find that miR-208a posttranscriptionally represses Thrap1, which is consistent with a previous report (17). We extended our previous observations by uncovering an additional conserved miR-208 family binding site within the Thrap1 3' UTR. Furthermore, we demonstrated that both miR-208a and miR-208b target those sites. In addition, we found that miR-208a and miR-208b target myostatin, a negative regulator of hypertrophic growth. The findings that both miR-208a and miR-208b repress Thrap1 and myostatin to a comparable level further suggest that miR-208 family members may target similar genes in vivo. A key disparity between our findings and that of an earlier report is the ability of miR-208a to induce cardiac hypertrophy. We believe the degree of miR-208a transgene expression accounts for this difference; we report higher miR-208a transgene expression levels, which indicate that surpassing a particular threshold of miR-208 family expression is important for facilitating hypertrophic growth.

In addition to the important role for miR-208a in cardiac hypertrophy, our studies also identify miR-208a as a key regulatory molecule necessary for proper cardiac conduction. Our experiments demonstrate that miR-208a is sufficient to induce cardiac arrhythmias, while miR-208a is also required to maintain proper cardiac conduction. Our results further indicate that miR-208a is required to maintain the expression of $\mathrm{Cx} 40$, whose misexpression is associated with cardiac arrhythmias (49). We also find that GATA4 and Hop are upregulated and downregulated, respectively, in Mir208a-/hearts. While GATA4 is directly targeted by miR-208a, Hop is likely indirectly regulated by miR-208a. We found that both transcript and protein levels of Hop were lower in $\mathrm{Mir}_{208 \mathrm{a}^{-/} \text {hearts compared }}$ with wild-type hearts. Thus, altering miR-208a expression affects the delicate regulation of potent cardiac transcription factors. However, the cardiac conduction defects revealed in miR-208a Tg and Mir208a $a^{-1-}$ mice did not phenocopy Cx40 and Hop loss-offunction animal models. This is perhaps not entirely surprising, since miRNAs are proposed to fine-tune the expression of targeted mRNAs that produce protein products important for a particular tissue and to reduce transcriptional noise by helping to turn over misexpressed mRNAs (68-73). By direct or indirect effects, a single miRNA may fine-tune the expression of thousands of genes (73). In this sense, the precise molecular mechanisms underlying miR-208amediated regulation of cardiac conduction system components have yet to be defined. Identification of the cardiac transcription factors whose expressions are posttranscriptionally fine-tuned by miR-208a will likely be the key to understanding miR-208a function. Additional studies are needed to test and further define this intriguing possibility.

Our present finding that miR-208a is required to maintain Hop expression may also help to explain the blunted hypertrophic growth response of Mir208a/- hearts (17). Unlike most homeobox transcription factors, Hop does not bind DNA directly. Instead, Hop recruits histone deacetylase 2 and inhibits the transcriptional activity of serum response factor in cardiomyocytes (74-76). Interestingly, Hop overexpression was reported to induce cardiac hypertrophy and is proposed to inhibit an antihypertrophy gene program in the adult heart (76). A potential explanation for the inability of Mir208a-/hearts to undergo hypertrophic growth may stem from the lack of Hop protein available to repress this antihypertrophy gene program. It will be important to understand how miR-208a regulates the expression of Hop and connexin proteins in the future.

Cardiac function and remodeling are intimately linked to the regulation of complex genetic pathways, and much effort has been expended in attempts to understand the molecular mechanisms underlying these pathways, with the ultimate goal of improving the prognosis of heart patients (77). Much of our current understanding of how cardiac gene expression is controlled is at the level of transcriptional regulation, in which transcription factors associate with their regulatory DNA elements (enhancer/ promoter sequences) to activate gene expression (78). The regulation of cardiac gene expression is complex, with individual cardiac genes controlled by multiple independent enhancers that direct very restricted expression patterns in the heart. miRNAs have reshaped our view of how cardiac gene expression is regulated, increasing this complexity even further, by adding another layer of regulation at the posttranscriptional level. The results reported in this study clearly established a role for miR-208a in repressing antihypertrophy genes as part of the genetic program needed for hypertrophic growth. Although the arrhythmogenesis induced by perturbing miR-208a levels warrants much caution, we anticipate that inhibition of miR-208a may be a viable therapeutic strategy to repress $\beta \mathrm{MHC}$ expression and might remove some of the maladaptive features of hypertrophy.

\section{Methods}

Generation of miR-208a Tg mice. All procedures were approved by and performed in accordance with the University of North Carolina Institutional Animal Care and Use Committee. A genomic fragment encoding the miR-208a precursor and flanking region was amplified by PCR using mouse genomic DNA as a template (miR-208a forward, GTCATCTAGAAAGCTTGATGCAGGAAAGAGCTTTGG, reverse, TGACAGATCTCAGCTGACATCCTCTAGGCTGGGGTT). This fragment was cloned into the pUHG10-3 tetracycline responsive vector plasmid at the $\mathrm{XbaI}$ site. A 2-kb fragment containing TRE, miR-208a precursor, and SV40 poly signal was excised by XhoI/AseI digestion and purified. The TRE-miR-208a gene was injected into the pronuclei of $\mathrm{C} 57 \mathrm{BL} / 6 \times \mathrm{C} 3 \mathrm{H}$ hybrid embryos and implanted into pseudo-pregnant recipient females by the University of North Carolina Animal Models Core. Five TREmiR-208 founder mice were established and crossed with C57BL/6 mice and expanded. Separate strains derived from 2 founders were maintained by mating animals heterozygous for TRE-miR-208a or $\alpha$ MHC-tTA, which expresses heart-specific tTA controlled by the heart-specific $\alpha$ MHC promoter (provided by Glenn I. Fishman, 
New York University, New York, New York, USA) (23). Single-Tg animals genotyped $\alpha \mathrm{MHC}$-tTA (referred to as "control mice") were compared with double-Tg littermates genotyped $\alpha$ MHC-tTA/ TRE-miR-208 (referred to as "miR-208a Tg mice").

Generation of Mir208a-/- mice. The miR-208a targeting vector was generated by digesting a $4.5-\mathrm{kb}$ fragment $\left(5^{\prime} \mathrm{arm}\right)$ with NheI/XhoI and ligated upstream of a positive-selection neomycin cassette flanked by loxP sites. A 1.8-kb fragment (3' arm) was digested with PmeI/NotI and ligated downstream of the neomycin cassette and upstream of a negative-selection thymidine kinase cassette. Targeted ES cells were identified by PCR and Southern blot analyses and used for blastocyst injection by the University of North Carolina Animal Models Core. The resulting chimeric mice were bred to $\mathrm{C} 57 \mathrm{BL} / 6$ mice to obtain germline transmission of the floxed allele. Subsequently, the neomycin cassette was excised by breeding with $\beta$-actin-Cre mice (79).

Pressure overload-induced hypertrophy model. Male C57BL/ 6 mice (6-8 weeks old) were subjected to pressure overload by thoracic aortic banding (25). Mice were sacrificed after 3 weeks of banding, and hearts were harvested for RNA extraction.

Hypothyroidism model. Thyroid hormone deficiency was induced by feeding adult mice for 4 weeks with iodine-free chow supplemented with 0.15\% PTU (catalog TD 97061; Harlan Teklad).

Histologic analysis of miR-208a Tg mice. Histologic analyses of heart tissues were performed according to standard procedures. Samples were stained with $\mathrm{H} \& \mathrm{E}$ for routine examination and agglutininwheat germ-TRITC conjugate to identify sarcolemmal membranes so that myofiber diameter could be quantified. Antibodies against desmin (catalog ab17156; Abcam) were used to visualize sarcomeric structure. Images were collected on an epifluorescent microscope (Eclipse E800; Nikon). Quantification of cardiomyocyte surface area was performed using ImageJ software on fluorescent micrographs from 4 hearts per genotype, using about 225 cells per heart across multiple sections.

Transthoracic echocardiography. Unanesthetized mice were restrained on a temperature-controlled mouse board (Indus Instruments), and echocardiography was performed on miR-208a Tg and control mice using a Vevo 660 ultrasound system (Visual Sonics) equipped with a $30-\mathrm{MHz}$ transducer. An echocardiographer blind to animal genotype captured 2-dimensional parasternal long axis views of the left ventricle. From this view, an M-mode cursor was positioned perpendicular to the interventricular septum and the posterior wall of the left ventricle at the level of the papillary muscles. The following measurements were obtained for systole and diastole using 4 cardiac cycles averaged: interventricular septal thickness, left ventricular posterior wall thickness, left ventricular internal diameter, heart rate, and fractional shortening.

Surface electrocardiograms. Mice were anesthetized with $1 \%-2 \%$ isoflurane in $700 \mathrm{ml} \mathrm{O}_{2} / \mathrm{min}$ via a face mask (following induction chamber containing $5 \%$ isoflurane). Rectal temperature was monitored and maintained at $37^{\circ} \mathrm{C}$ using a heat pad and heat lamp. ECGs using multiple leads were recorded for about 3 minutes at 2 $\mathrm{MHz}$ from needle electrodes inserted subcutaneously into the each limb. Recordings were analyzed using the ECG module of LabChart 5 software (AD Instruments), and QTc intervals were calculated using a formula developed for analyzing murine data (80).

Northern blot analysis and RT-PCR. RNA analyses by miRNA Northern blot, semi-quantitative RT-PCR, and quantitative RT-PCR were essentially performed as described $(12,31,81)$. Quantitative RT-PCR was performed on 5 mouse hearts per genotype tested.
Immunoblotting and immunostaining. Immunoblotting and immunostaining were essentially performed as previously described (12) using antibodies to $\beta$ MHC (catalog M8421; Sigma-Aldrich), MHC (antibody MF20; University of Iowa Developmental Studies Hybridoma Bank), myostatin (catalog AB3239; Chemicon, Millipore), Thrap1 (a gift from Robert G. Roeder, Rockefeller University, New York, New York, USA), Cx40 (catalog 36-4900; Invitrogen), Cx43 (catalog C8093; Sigma-Aldrich), GATA4 (catalog sc-1237; Santa Cruz Biotechnology Inc.), Hop (a gift from Eric Olson, University of Texas Southwestern Medical Center at Dallas, Dallas, Texas, USA), $\beta$-tubulin (catalog C4585; Sigma-Aldrich), and GAPDH (catalog MAB374; Chemicon, Millipore). Western blotting was performed on least 4 animals per genotype and repeated independently.

miRNA in situ bybridization. miRNA in situ hybridization was performed essentially as previously described (82) using locked nucleic acid (LNA) probes complementary to mouse mature miR-208 (product 39102-05; Exiqon) or scrambled control sequence (product 99004-05; Exiqon) with 3' digoxigenin conjugates. LNA probes were hybridized to mouse heart cryosections and washed at $37^{\circ} \mathrm{C}$.

Cultured cardiomyocyte experiments. Preparation of neonatal rat cardiomyocytes was done as previously described (81). Cardiomyocytes were treated with triiodo-L-thyronine (Sigma-Aldrich) essentially as previously described (83). Antisense 2'O-methylmodified oligonucleotides were transfected using Lipofectamine (Invitrogen). Cardiomyocytes were transduced with miR-208a or GFP-expressing adenoviruses at MOI 10. Immunostaining performed using antibodies against $\alpha$-actinin (catalog A5044; SigmaAldrich), $\beta$ MHC (catalog M8421; Sigma-Aldrich), and ANF (catalog sc-20158; Santa Cruz Biotechnology Inc.). For fluorescence intensity analysis, individual cardiomyocytes (at least 100 cell bodies per condition) were measured on a $0-255$ grayscale using ImageJ software. The intensity of immunostaining was reported as the fold change in mean gray value \pm SEM. Cardiomyocyte experiments were independently repeated at least 3 times.

Confocal analysis of hearts from $m i R-208 a \mathrm{Tg}$; YFP- $\beta$ MHC mice. The miR-208a $\mathrm{Tg}$ line was bred with the YFP- $\beta$ MHC reporter line. The YFP- $\beta$ MHC mice harbor a YFP- $\beta$ MHC fusion allele that allows precise and accurate assessment of $\beta \mathrm{MHC}$ expression in adult hearts (31). Hearts from heterozygous triple-Tg mice expressing

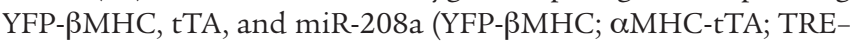
miR-208a mice) were compared with heterozygous double-Tg littermates expressing YFP- $\beta$ MHC and tTA (YFP- $\beta$ MHC; $\alpha M H C-t T A$ mice). Paraformaldehyde-fixed hearts were sectioned with a vibrotome at $150-\mu \mathrm{m}$ thicknesses. Individual sections were treated with sodium borohydride ( $1 \mathrm{mg} / \mathrm{ml}$ in PBS) for 30 minutes to reduce fixative-induced fluorescence. Sections were stained with Alexa Fluor 633-conjugated agglutinin wheat germ and analyzed with a FV500 confocal microscope (Olympus). Morphometric analyses were conducted on coronal sections using individual cell areas from the left ventricular free wall that were traced with ImageJ software (31).

Cloning and expression of miR-208a, miR-208b, and miR-124. Genomic fragments of miR-208a, miR-208b, and miR-124 precursors were amplified by PCR using mouse genomic DNA as a template. PCR products were cloned into pcDNA(+)3.1 vector (Invitrogen), and miRNA expression was confirmed by Northern blot analysis of transfected 293T cells (Fugene6; Roche).

Luciferase reporter assays. A modified pGL3-control vector (pGL3cm) for 3' UTR-luciferase reporter assays was described previously (12). The TargetScanHuman database (http://www. 
targetscan.org/) was used to identify predicted miR-208 binding sites. A 575-bp fragment of the Thrap1 3' UTR was amplified from a mouse cDNA library and cloned downstream of the luciferase gene to create the luc-Thrap1 construct. Duplication of the 2 Thrap 1 target sites was accomplished by PCR subcloning a portion of the Thrap1 3' UTR directly into the initial Thrap1 construct to create luc-Thrap1 $4 \times$. Seed region mutations were generated by site-directed mutagenesis (Supplemental Figure 6). The luc-myostatin $4 \times$ and luc-GATA4 $4 \times$ reporter constructs were generated by annealing oligonucleotides encoding 2 mouse miR-208 target sites separated by $10 \mathrm{bp}$ and cloning them in tandem downstream of the luciferase gene. To confirm miRNA expression in the reporter assays, we generated miRNA sensor constructs consisting of perfectly complementary sequences to miR-208a or miR-124 directly downstream of the luciferase gene. Reporter assays were conducted using 293T cells in triplicate at least 3 times in 24-well plates. Transfections were performed with $50 \mathrm{ng}$ of reporter and 50,100 , or 150 ng of miRNA plasmids (Fugene6; Roche). A CMVlacZ reporter was used as an internal control to normalize for transfection efficiencies, and the total amount of DNA per well was kept constant by adding the corresponding amount of empty expression vector.
Statistics. Values are reported as means \pm SEM unless indicated otherwise. The 2-tailed Mann-Whitney $U$ test was used for comparing 2 means (Prism; GraphPad). Values of $P<0.05$ were considered statistically significant.

\section{Acknowledgments}

We thank Xiaoyun Hu for excellent technical support and Oliver Smithies for comments. This work was supported by the March of Dimes Birth Defect Foundation, NIH, American Heart Association, and Muscular Dystrophy Association (grant to D.-Z. Wang). Z. Deng is supported by Chinese National Natural Science Foundation grant 30772211. T.E. Callis is a Predoctoral Fellow, K. Pandya is a Postdoctoral Fellow, and D.-Z. Wang is an Established Investigator of the American Heart Association.

Received for publication May 9, 2008, and accepted in revised form June 10, 2009.

Address correspondence to: Da-Zhi Wang, Department of Cardiology, Children's Hospital Boston, Harvard Medical School, 320 Longwood Avenue, Boston, Massachusetts 02115, USA. Phone: (617) 9194768; Fax: (617) 731-0787; E-mail: dwang@enders.tch.harvard.edu.
1. Filipowicz, W., Bhattacharyya, S.N., and Sonenberg, N. 2008. Mechanisms of post-transcriptional regulation by microRNAs: are the answers in sight? Nat. Rev. Genet. 9:102-114.

2. Kiriakidou, M., et al. 2007. An mRNA m7G cap binding-like motif within human Ago2 represses translation. Cell. 129:1141-1151.

3. Bagga, S., et al. 2005. Regulation by let-7 and lin-4 miRNAs results in target mRNA degradation. Cell. 122:553-563.

4. Humphreys, D.T., Westman, B.J., Martin, D.I., and Preiss, T. 2005. MicroRNAs control translation initiation by inhibiting eukaryotic initiation factor 4E/cap and poly(A) tail function. Proc. Natl. Acad. Sci. U. S. A. 102:16961-16966.

5. Callis, T.E., Deng, Z., Chen, J.F., and Wang, D.Z. 2008. Muscling through the microRNA world. Exp. Biol. Med. (Maywood). 233:131-138.

6. Ambros, V. 2004. The functions of animal microRNAs. Nature. 431:350-355.

7. Kloosterman, W.P., and Plasterk, R.H. 2006. The diverse functions of microRNAs in animal development and disease. Dev. Cell. 11:441-450.

8. Callis, T.E., and Wang, D.Z. 2008. Taking microRNAs to heart. Trends Mol. Med. 14:254-260.

9. van Rooij,E.,Liu,N., and Olson,E.N.2008. MicroRNAs flex their muscles. Trends Genet. 24:159-166.

10. Chen, J.F., Callis, T.E., and Wang, D.Z. 2009. microRNAs and muscle disorders. J. Cell. Sci. 122:13-20.

11. Zhao, Y., Samal, E., and Srivastava, D. 2005. Serum response factor regulates a muscle-specific microRNA that targets Hand 2 during cardiogenesis. Nature. 436:214-220.

12. Chen, J.F., et al. 2006. The role of microRNA-1 and microRNA-133 in skeletal muscle proliferation and differentiation. Nat. Genet. 38:228-233.

13. Zhao, Y., et al. 2007. Dysregulation of cardiogenesis, cardiac conduction, and cell cycle in mice lacking miRNA-1-2. Cell. 129:303-317.

14. Chen, J.F., et al. 2008. Targeted deletion of Dicer in the heart leads to dilated cardiomyopathy and heart failure. Proc. Natl. Acad. Sci. U. S. A. 105:2111-2116.

15. Thum, T., et al. 2007. MicroRNAs in the human heart: a clue to fetal gene reprogramming in heart failure. Circulation. 116:258-267.

16. van Rooij, E., et al. 2006. A signature pattern of stress-responsive microRNAs that can evoke car- diac hypertrophy and heart failure. Proc. Natl. Acad. Sci.U.S. A. 103:18255-18260.

17. van Rooij, E., et al. 2007. Control of stress-dependent cardiac growth and gene expression by a microRNA. Science. 316:575-579.

18. Lompre, A.M., Nadal-Ginard, B., and Mahdavi, V. 1984. Expression of the cardiac ventricular alphaand beta-myosin heavy chain genes is developmentally and hormonally regulated. J. Biol. Chem. 259:6437-6446.

19. Chien, K.R. 2000. Genomic circuits and the integrative biology of cardiac diseases. Nature. 407:227-232

20. Frey, N., Katus, H.A., Olson, E.N., and Hill, J.A. 2004. Hypertrophy of the heart: a new therapeutic target? Circulation. 109:1580-1589.

21. Ventura, A., et al. 2008. Targeted deletion reveals essential and overlapping functions of the miR-17 through 92 family of miRNA clusters. Cell. 132:875-886.

22. Gupta, M.P. 2007. Factors controlling cardiac myosin-isoform shift during hypertrophy and heart failure. J. Mol. Cell. Cardiol. 43:388-403.

23. Passman, R.S., and Fishman, G.I. 1994. Regulated expression of foreign genes in vivo after germline transfer. J. Clin. Invest. 94:2421-2425.

24. Braunwald, E., and Bristow, M.R. 2000. Congestive heart failure: fifty years of progress. Circulation. 102:IV14-IV23.

25. Tatsuguchi, M., et al. 2007. Expression of microRNAs is dynamically regulated during cardiomyocyte hypertrophy. J. Mol. Cell. Cardiol. 42:1137-1141.

26. Cheng, Y., et al. 2007. MicroRNAs are aberrantly expressed in hypertrophic heart: do they play a role in cardiac hypertrophy? Am. J. Pathol. 170:1831-1840.

27. Sayed, D., Hong, C., Chen, I.Y., Lypowy, J., and Abdellatif, M. 2007. MicroRNAs play an essential role in the development of cardiac hypertrophy. Circ. Res. 100:416-424.

28. Krenz, M., et al. 2003. Analysis of myosin heavy chain functionality in the heart. J. Biol. Chem. 278:17466-17474.

29. Tardiff, J.C., et al. 2000. Expression of the beta (slow)-isoform of MHC in the adult mouse heart causes dominant-negative functional effects. Am. J. Physiol. Heart Circ. Physiol. 278:H412-H419.

30. Krenz, M., and Robbins, J. 2004. Impact of beta- myosin heavy chain expression on cardiac function during stress. J. Am. Coll. Cardiol. 44:2390-2397.

31. Pandya, K., Kim, H.S., and Smithies, O. 2006. Fibrosis, not cell size, delineates beta-myosin heavy chain reexpression during cardiac hypertrophy and normal aging in vivo. Proc. Natl. Acad. Sci. U. S. A. 103:16864-16869.

32. Grimson, A., et al. 2007. MicroRNA targeting specificity in mammals: determinants beyond seed pairing. Mol. Cell. 27:91-105.

33. Lewis, B.P., Burge, C.B., and Bartel, D.P. 2005. Conserved seed pairing, often flanked by adenosines, indicates that thousands of human genes are microRNA targets. Cell. 120:15-20.

34. Morkin, E. 1993. Regulation of myosin heavy chain genes in the heart. Circulation. 87:1451-1460.

35. Morkin, E. 2000. Control of cardiac myosin heavy chain gene expression. Microsc. Res. Tech. 50:522-531.

36. Lee, S.J. 2004. Regulation of muscle mass by myostatin. Annu Rev Cell Dev. Biol. 20:61-86.

37. Schuelke, M., et al. 2004. Myostatin mutation associated with gross muscle hypertrophy in a child. N. Engl. J. Med. 350:2682-2688.

38. Morissette, M.R., et al. 2006. Myostatin regulates cardiomyocyte growth through modulation of Akt signaling. Circ. Res. 99:15-24.

39. Cook, S.A., Matsui, T., Li, L., and Rosenzweig, A. 2002. Transcriptional effects of chronic Akt activation in the heart. J. Biol. Chem. 277:22528-22533.

40. Shyu, K.G., Lu, M.J., Wang, B.W., Sun, H.Y., and Chang, H. 2006. Myostatin expression in ventricular myocardium in a rat model of volume-overload heart failure. Eur. J. Clin. Invest. 36:713-719.

41. Shyu, K.G., Ko, W.H., Yang, W.S., Wang, B.W., and Kuan, P. 2005. Insulin-like growth factor-1 mediates stretch-induced upregulation of myostatin expression in neonatal rat cardiomyocytes. Cardiovasc. Res. 68:405-414.

42. Ito, M., et al. 1999. Identity between TRAP and SMCC complexes indicates novel pathways for the function of nuclear receptors and diverse mammalian activators. Mol. Cell. 3:361-370.

43. Wang, J.C., Walker, A., Blackwell, T.K., and Yamamoto, K.R. 2004. The Caenorhabditis elegans ortholog of TRAP240, CeTRAP240/let-19, selectively modulates gene expression and is essential for embryogenesis. J. Biol. Chem. 279:29270-29277. 44. Dupont, E., et al. 2001. Altered connexin expres- 
sion in human congestive heart failure. J. Mol. Cell. Cardiol. 33:359-371.

45. Kaprielian, R.R., et al. 1998. Downregulation of immunodetectable connexin43 and decreased gap junction size in the pathogenesis of chronic hibernation in the human left ventricle. Circulation. 97:651-660.

46. Kostin, S., et al. 2003. Gap junction remodeling and altered connexin 43 expression in the failing human heart. Mol. Cell. Biochem. 242:135-144.

47. Sepp, R., Severs, N.J., and Gourdie, R.G. 1996. Altered patterns of cardiac intercellular junction distribution in hypertrophic cardiomyopathy. Heart. 76:412-417.

48. Lo, C.W. 2000. Role of gap junctions in cardiac conduction and development: insights from the connexin knockout mice. Circ. Res. 87:346-348.

49. Simon, A.M., Goodenough, D.A., and Paul, D.L. 1998. Mice lacking connexin 40 have cardiac conduction abnormalities characteristic of atrioventricular block and bundle branch block. Curr. Biol. 8:295-298.

50. Guerrero, P.A., et al. 1997. Slow ventricular conduction in mice heterozygous for a connexin 43 null mutation. J. Clin. Invest. 99:1991-1998.

51. Gros, D.B., and Jongsma, H.J. 1996. Connexins in mammalian heart function. Bioessays. 18:719-730.

52. Ismat, F.A., et al. 2005. Homeobox protein Hop functions in the adult cardiac conduction system. Circ. Res. 96:898-903.

53. Takebayashi-Suzuki, K., Pauliks, L.B., Eltsefon, Y. and Mikawa, T. 2001. Purkinje fibers of the avian heart express a myogenic transcription factor program distinct from cardiac and skeletal muscle. Dev. Biol. 234:390-401.

54. Linhares, V.L., et al. 2004. Transcriptional regulation of the murine Connexin 40 promoter by cardiac factors Nkx2-5, GATA4 and Tbx5. Cardiovasc. Res. 64:402-411.

55. Griffiths-Jones, S., Saini, H.K., van Dongen, S., and Enright, A.J. 2008. miRBase: tools for microRNA genomics. Nucleic Acids Res. 36:D154-D158.

56. Weiss, A., and Leinwand, L.A. 1996. The mammalian myosin heavy chain gene family. Annu. Rev. Cell Dev. Biol. 12:417-439.

57. VanBuren, P., Harris, D.E., Alpert, N.R., and War- shaw, D.M. 1995. Cardiac V1 and V3 myosins differ in their hydrolytic and mechanical activities in vitro. Circ. Res. 77:439-444.

58. Patel, R., et al. 2001. Simvastatin induces regression of cardiac hypertrophy and fibrosis and improves cardiac function in a transgenic rabbit model of human hypertrophic cardiomyopathy. Circulation. 104:317-324

59. Abraham, W.T., et al. 2002. Coordinate changes in Myosin heavy chain isoform gene expression are selectively associated with alterations in dilated cardiomyopathy phenotype. Mol. Med. 8:750-760.

60. Lowes, B.D., et al. 2002. Myocardial gene expression in dilated cardiomyopathy treated with beta-blocking agents. N. Engl. J. Med. 346:1357-1365.

61. Yasumura, Y., Takemura, K., Sakamoto, A., Kitakaze, M., and Miyatake, K. 2003. Changes in myocardial gene expression associated with beta-blocker therapy in patients with chronic heart failure. J. Card. Fail. 9:469-474.

62. Stansfield, W.E., et al. 2008. Proteasome inhibition promotes regression of left ventricular hypertrophy. Am. J. Physiol. Heart Circ. Physiol. 294:H645-H650.

63. Ahmad, F., Seidman, J.G., and Seidman, C.E. 2005. The genetic basis for cardiac remodeling. Annu. Rev. Genomics Hum. Genet. 6:185-216.

64. Kuzman, J.A., Vogelsang, K.A., Thomas, T.A., and Gerdes, A.M. 2005. L-Thyroxine activates Akt signaling in the heart. J. Mol. Cell. Cardiol. 39:251-258.

65. Degens, H., et al. 2003. Functional and metabolic adaptation of the heart to prolonged thyroid hormone treatment. Am. J. Physiol. Heart Circ. Physiol. 284:H108-H115.

66. Chang, K.C., et al. 1997. Thyroid hormone improves function and $\mathrm{Ca} 2+$ handling in pressure overload hypertrophy. Association with increased sarcoplasmic reticulum Ca2+-ATPase and alpha-myosin heavy chain in rat hearts. J. Clin. Invest. 100:1742-1749.

67. Ching, G.W., et al. 1996. Cardiac hypertrophy as a result of long-term thyroxine therapy and thyrotoxicosis. Heart. 75:363-368.

68. Hornstein, E., and Shomron, N. 2006. Canalization of development by microRNAs. Nat. Genet. 38(Suppl.):S20-S24.

69. Flynt, A.S., Li, N., Thatcher, E.J., Solnica-Krezel, L. and Patton, J.G. 2007. Zebrafish miR-214 modu- lates Hedgehog signaling to specify muscle cell fate. Nat. Genet. 39:259-263.

70. Giraldez, A.J., et al. 2005. MicroRNAs regulate brain morphogenesis in zebrafish. Science. 308:833-838.

71. Boutz, P.L., Chawla, G., Stoilov, P., and Black, D.L. 2007. MicroRNAs regulate the expression of the alternative splicing factor $\mathrm{nPTB}$ during muscle development. Genes Dev. 21:71-84.

72. Baek, D., et al. 2008. The impact of microRNAs on protein output. Nature. 455:64-71.

73. Selbach, M., et al. 2008. Widespread changes in protein synthesis induced by microRNAs. Nature. 455:58-63.

74. Chen, F., et al. 2002. Hop is an unusual homeobox gene that modulates cardiac development. Cell. 110:713-723.

75. Shin, C.H., et al. 2002. Modulation of cardiac growth and development by HOP, an unusual homeodomain protein. Cell. 110:725-735.

76. Kook, H., et al. 2003. Cardiac hypertrophy and histone deacetylase-dependent transcriptional repression mediated by the atypical homeodomain protein Hop. J. Clin. Invest. 112:863-871.

77. Olson, E.N. 2004. A decade of discoveries in cardiac biology. Nat. Med. 10:467-474.

78. Olson, E.N. 2006. Gene regulatory networks in the evolution and development of the heart. Science. 313:1922-1927.

79. Lewandoski, M., Meyers, E.N., and Martin, G.R. 1997. Analysis of Fgf8 gene function in vertebrate development. Cold Spring Harb. Symp. Quant. Biol. 62:159-168.

80. Mitchell, G.F., Jeron, A., and Koren, G. 1998. Measurement of heart rate and Q-T interval in the conscious mouse. Am. J. Physiol. 274:H747-H751.

81. Callis, T.E., Cao, D., and Wang, D.Z. 2005. Bone morphogenetic protein signaling modulates myocardin transactivation of cardiac genes. Circ. Res. 97:992-1000.

82. Obernosterer, G., Martinez, J., and Alenius, M. 2007. Locked nucleic acid-based in situ detection of microRNAs in mouse tissue sections. Nat. Protoc. 2:1508-1514.

83. Williams, H.M., and Ianuzzo, C.D. 1988. The effects of triiodothyronine on cultured neonatal rat cardiac myocytes. J. Mol. Cell. Cardiol. 20:689-699. 